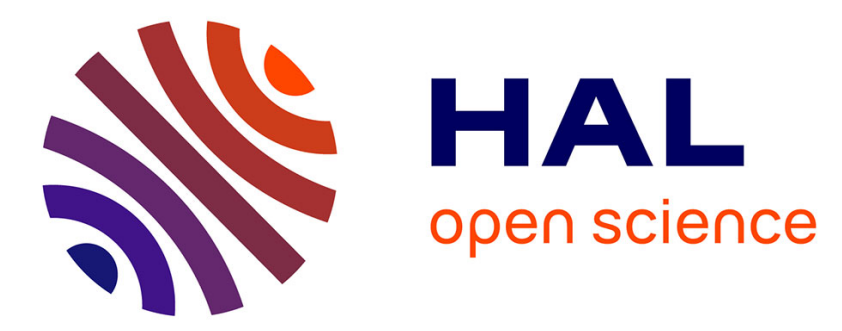

\title{
Étude des aberrations du second ordre du microanalyseur ionique et de quelques autres systèmes destinés au filtrage d'images étendues
}

\author{
M. Leleyter, G. Slodzian
}

\section{- To cite this version:}

M. Leleyter, G. Slodzian. Étude des aberrations du second ordre du microanalyseur ionique et de quelques autres systèmes destinés au filtrage d'images étendues. Revue de Physique Appliquée, 1971, 6 (1), pp.65-89. 10.1051/rphysap:019710060106500 . jpa-00243505

\section{HAL Id: jpa-00243505 https://hal.science/jpa-00243505}

Submitted on 1 Jan 1971

HAL is a multi-disciplinary open access archive for the deposit and dissemination of scientific research documents, whether they are published or not. The documents may come from teaching and research institutions in France or abroad, or from public or private research centers.
L'archive ouverte pluridisciplinaire HAL, est destinée au dépôt et à la diffusion de documents scientifiques de niveau recherche, publiés ou non, émanant des établissements d'enseignement et de recherche français ou étrangers, des laboratoires publics ou privés. 


\title{
ÉTUDE DES ABERRATIONS DU SECOND ORDRE DU MICROANALYSEUR IONIQUE ET DE QUELQUES AUTRES SYSTẼMES DESTINÉS AU FILTRAGE D'IMAGES ÉTENDUES
}

\author{
M. LELEYTER (Mme) $\left(^{*}\right)$ \\ et G. SLODZIAN \\ Faculté des Sciences, 33, rue Saint-Leu, Amiens, Somme \\ Laboratoire de Physique des Solides associé au C. N. R. S., Bâtiment 510, \\ Faculté des Sciences d'Orsay, Essonne \\ (Reçu le 10 juillet 1970, révisé le 27 octobre 1970)
}

\begin{abstract}
Résumé. - On calcule géométriquement les aberrations du second ordre entachant les images d'objets étendus donnés par quelques systèmes dispersifs, notamment, celles du microanalyseur ionique constitué par un double prisme magnétique à champ uniforme associé à un miroir électrostatique. Les aberrations du second ordre les plus importantes sont les aberrations géométriques de champ et les aberrations chromatiques. On cherche à les minimiser en déterminant par le calcul la meilleure position de l'objet. Dans le cas du microanalyseur ionique, on trouve que l'aberration totale, ramenée au plan de l'objet, est de l'ordre de $0,3 \mu$ sur les bords d'un champ image de $200 \mu$ lorsque la lentille à émission est munie d'un diaphragme de contraste de $400 \mu$ qui permet d'obtenir une limite de résolution de $1 \mu$.
\end{abstract}

\begin{abstract}
By geometrical means we have computed the second-order aberrations on the images of broad objects given by several dispersive systems, specially those occurring in the ion microanalyzer which is composed by a double homogeneous magnetic prism associated with an electrostatic mirror. The most important second-order aberrations are field-astigmatism and chromatic aberrations. It has been found that the minimum aberration was reached at a given position of the object. In the case of the ion microanalyzer, from our estimations, the overall aberration at that position is about $0.3 \mu$ at the edge of a $200 \mu$-field in the object space when the emission lens is apertured with a $400 \mu$-diaphragm which allows a resolving limit of $1 \mu$.
\end{abstract}

Introduction. - Les systèmes dispersifs sont utilisés couramment en Optique corpusculaire pour trier les particules chargées selon leur énergie ou leur masse. Divers montages expérimentaux [1 à 7] ont ajouté à cette fonction de filtrage la possibilité de conserver l'image d'une région étendue de l'objet initialement donnée par une lentille. On obtient ainsi des images qui ne sont formées que par des corpuscules d'un type déterminé ou par des particules dont l'énergie est comprise dans une bande donnée. Naturellement, il est essentiel que le filtrage n'altère pas la qualité de l'image initiale et par suite, il est indispensable d'examiner de près les aberrations produites par l'optique non gaussienne des systèmes dispersifs.

Nous nous sommes intéressés plus particulièrement à l'optique de filtrage du microanalyseur ionique [8] constituée par l'association d'un miroir électrostatique et d'un double prisme magnétique à induction uni-

(*) Cet article constitue la première partie d'une thèse de Doctorat d'Etat ès Sciences physiques qui sera soutenue à la Faculté des Sciences d'Orsay. forme. Mais au passage, nous avons également calculé les aberrations du dispositif utilisé par Castaing et Henry [5] pour opérer un filtrage des vitesses en Microscopie électronique ainsi que les défauts introduits sur les images ioniques par une simple déflexion magnétique [3, 4]. Les calculs que nous présentons ici constituent une première approximation dans laquelle le miroir est supposé dépourvu d'aberration et où l'on admet que l'induction magnétique s'arrête brusquement à l'aplomb des faces polaires.

La méthode utilisée consiste à faire le calcul analytique des trajectoires au troisième ordre près en portant une attention particulière aux effets combinés de la discontinuité de l'induction et de l'inclinaison des faces polaires par rapport à l'axe moyen du faisceau ; ces effets provoquent non seulement la convergence des trajectoires situées hors du plan de symétrie, mais aussi l'apparition de nouveaux termes d'aberrations. Cette méthode est moins générale que celle développée par Cotte [9] sur la base d'un formálisme hamiltonien, mais dans le cas des faces magnétiques inclinées, elle conduit à des calculs plus simples dont on peut avoir 
à chaque étape une représentation physique immédiate.

Dans le cas de l'analyseur ionique par exemple, une lentille à émission donne de la surface d'un objet bombardé par des ions primaires une image agrandie I composée par tous les ions secondaires arrachés au cours de la pulvérisation. L'image I sert d'objet pour le système dispersif constitué par l'association du double prisme magnétique et du miroir électrostatique. Les ions secondaires sont une première fois déviés de $90^{\circ}$ par le prisme, puis rencontrent le miroir où les plus rapides sont éliminés lorsqu'ils touchent l'électrode de contre-champ, et les plus lents réfléchis ; ces particules réfléchies sont ainsi renvoyées dans le prisme où elles subissent une seconde déflexion de $90^{\circ}$, mais en sens inverse de la première, de sorte que l'axe moyen du faisceau émergent est dans le prolongement de l'axe moyen du faisceau incident. L'image I est transformée en une image virtuelle I" entachée d'aberrations ; cependant celles-ci affectent une image agrandie, de sorte que, ramenées à l'objet, leur influence sur la résolution est notablement réduite.

Selon la disposition des divers éléments optiques (centre et sommet du miroir, couples de points stigmatiques et focale achromatique du prisme), le système peut être rendu stigmatique et achromatique au niveau de $I^{\prime \prime}$ pour une position donnée de I, ou astigmatique, mais complètement achromatique après la deuxième traversée quelle que soit la position de I. Cette dernière disposition est celle adoptée dans le microanalyseur car elle permet d'utiliser le miroir comme « filtre passe-bas » pour les énergies indépendamment de la nature des ions.

On a cherché à déterminer dans ces diverses dispositions comment variaient les aberrations dans le plan image $I^{\prime \prime}$ avec la position de I pour savoir s'il était possible de les minimiser.

I. Rappel des propriétés du premier ordre du système optique à l'approximation de Gauss. - Considérons un faisceau de trajectoires parcourues par des ions identiques et monocinétiques, issu d'un point $\mathrm{C}$, et dont l'axe moyen est contenu dans le plan de symétrie du prisme. L'axe de ce faisceau attaque la face d'entrée du prisme magnétique sous l'incidence $\varepsilon$ et émerge perpendiculairement à la face de sortie après avoir subi une déviation de $\Phi=\pi / 2$ (Fig. 1). La trajectoire moyenne du faisceau reste dans le plan de symétrie (première section principale) et constitue l'axe principal du système optique. Les trajectoires issues de $\mathrm{C}$, dont les vitesses initiales sont contenues dans le plan de symétrie, restent dans ce plan, et, après rotation dans l'entrefer, vont converger, au deuxième ordre près, en un point $\mathbf{C}_{1}^{\prime}$ de la trajectoire principale.

Les trajectoires issues de $\mathrm{C}$, dont les vitesses initiales sont contenues dans un plan perpendiculaire au plan de symétrie, restent dans une surface cylindrique (deuxième section principale) dont les génératrices, perpendiculaires au plan radial, s'appuient sur l'axe

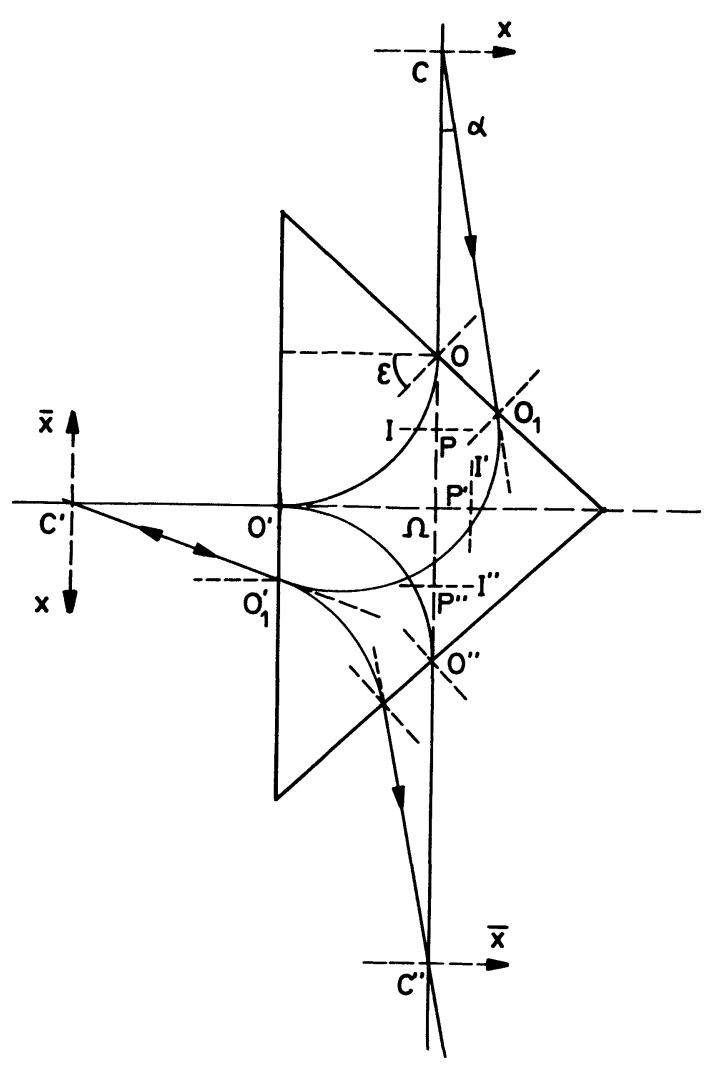

FIG. 1. - Trajectoire au premier ordre dans le plan radial d'une particule issue de $\mathrm{C}$, faisant l'angle $\alpha$ avec l'axe $\mathrm{CO}$ (cas du système stigmatique : I : trace du plan objet; l'induction $\mathbf{B}_{0}$ est perpendiculaire au plan de figure.

- $1^{\text {re }}$ traversée du prisme (axe $\mathrm{x}$ normal à l'axe optique). $I^{\prime}$ trace du plan image de I (au premier ordre); $C$ et $C^{\prime}$ points stigmatiques réels, $\mathbf{P}$ et $\mathbf{P}^{\prime}$ points stigmatiques virtuels.

$-2^{\mathrm{e}}$ traversée du prisme (axe $\bar{x}$ normal à l'axe optique) : $\mathrm{I}^{\prime \prime}$ : trace du plan image de $\mathbf{I}^{\prime}$ (au premier ordre); $\mathbf{C}^{\prime}$ et $\mathbf{C}^{\prime \prime}$ points stigmatiques réels, $\mathrm{P}^{\prime}$ et $\mathrm{P}^{\prime \prime}$ points stigmatiques virtuels.

principal. A la sortie de l'aimant, elles vont converger, au deuxième ordre près, en un point $C_{2}^{\prime}$ de l'axe.

Après rotation dans l'aimant, l'ensemble des trajectoires issues de $\mathrm{C}$ et situées dans un cône de révolution de demi-angle au sommet $\alpha$, s'appuieront, au second ordre près en $\alpha$, sur deux focales, l'une radiale, parallèle à l'induction magnétique $\mathbf{B}_{0}$, passant par $\mathbf{C}_{1}^{\prime}$, l'autre axiale, perpendiculaire à $\mathbf{B}_{0}$ et passant par $\mathbf{C}_{2}^{\prime}$.

I.1 PoInTs STIGMATIQUES. - I.1.1 Première traversée. - Si $\mathrm{O}$ est le point d'entrée dans l'aimant de la trajectoire principale et $\mathrm{O}^{\prime}$ le point de sortie, on définit la position d'un point objet $\mathbf{M}$ par son abscisse réduite $l$ sur l'axe $\mathrm{CO}$ orienté dans le sens du mouvement avec $l=\overline{\mathrm{OM}} / R_{0}$, où $R_{0}$ est le rayon de la trajectoire principale dans l'entrefer ( $l$ est négative pour les objets réels).

Après rotation dans la première traversée, un pinceau de trajectoires issu du point objet $\mathbf{M}$ (ou allant converger en $\mathbf{M}$ ) situé sur l'axe sera transformé en un pinceau s'appuyant sur deux focales, l'une radiale en $\mathrm{M}_{\mathrm{r}}^{\prime}$, l'autre axiale en $\mathrm{M}_{\mathrm{a}}^{\prime}$ telles que $\overline{\mathrm{O}^{\prime} \mathrm{M}_{\mathrm{r}}^{\prime}}=l_{r}^{\prime} R_{0}$ et 
$\overline{\mathrm{O}^{\prime}} \overline{\mathrm{M}_{\mathrm{a}}^{\prime}}=l_{\mathrm{a}}^{\prime} R_{0}$ (les $l^{\prime}$ sont positifs pour les images réelles).

L'étude faite par Henry $[5,6]$ à partir des calculs de Cotte [9], de même que les résultats de Cross [10], montrent que le prisme possède deux couples de points stigmatiques conjugués - c'est-à-dire pour lesquels $l_{\mathrm{r}}^{\prime}=l_{\mathrm{a}}^{\prime}=l^{\prime}-$ si l'on choisit pour $\operatorname{tg} \varepsilon=t$ une valeur prise hors de l'intervalle ] $-1 \pi / 4,1-\pi / 4[$. De plus, le calcul montre que si l'on veut placer l'un des points d'un couple stigmatique en $\mathrm{C}$, à $2 R_{0}$ en avant de la face d'entrée du prisme $\left(\overrightarrow{\mathrm{OC}}=-2 R_{0}\right)$, il faut que $\operatorname{tg} \varepsilon$ soit égale à 0,843 . Les positions des points stigmatiques sont alors les suivantes :

couple de points réels

$$
\left\{\begin{aligned}
\overline{\mathrm{OC}} & =l_{1} R_{0}=-2 R_{0} \\
\overline{\mathrm{O}^{\prime} \mathrm{C}^{\prime}} & =l_{1}^{\prime} R_{0}=R_{0}\left(t+\frac{1}{2}\right)=1,343 R_{0}
\end{aligned}\right.
$$

couple de points virtuels

$$
\left\{\begin{array}{l}
\overline{\mathrm{OP}}=l_{2} R_{0}=\frac{2 R_{0}}{\pi+1}=0,483 R_{0} \\
\overline{\mathrm{O}^{\prime} \mathrm{P}^{\prime}}=l_{2}^{\prime} R_{0}=R_{0}\left(t-\frac{\pi+1}{2}\right)=1,228 R_{0} .
\end{array}\right.
$$

I.1.2 Deuxième traversée du prisme. - Considérons un faisceau de particules identiques aux précédentes dont l'axe coïncide avec la trajectoire principale à la sortie de la première traversée et qui attaque maintenant la face d'entrée du prisme sous l'incidence normale (cet axe est un axe de symétrie pour la figure). A l'entrée de cette deuxième traversée, la vitesse des particules a changé de sens; il en est donc de même de la force magnétique; la trajectoire principale dans le prisme est un arc de cercle symétrique du précédent par rapport à l'axe de symétrie de la figure (Fig. 1). On appelle $\bar{\varepsilon}^{\prime}$ l'angle de sortie, et par construction, on a $\bar{\varepsilon}^{\prime}=\varepsilon$.

Pour $\operatorname{tg} \varepsilon=0,843$, les positions des points stigmatiques sont (on distinguera désormais les grandeurs relatives à la seconde traversée en les surmontant d'un trait) :

couple de points réels

$$
\left\{\begin{array}{l}
\overline{\mathrm{O}^{\prime} \mathrm{C}_{1}^{\prime}}=\bar{l}_{1} R_{0}=-R_{0}\left(t+\frac{1}{2}\right) \\
\overline{\mathrm{O}^{\prime \prime} \mathrm{C}^{\prime \prime}}=\bar{l}_{1}^{\prime} R_{0}=2 R_{0}
\end{array}\right.
$$

couple de points virtuels

$$
\left\{\begin{array}{l}
\overline{\mathrm{O}^{\prime} \mathrm{P}_{1}^{\prime}}=\bar{l}_{2} R_{0}=-R_{0}\left(t-\frac{\pi+{ }^{\prime \prime}}{2}\right) \\
\overline{\mathrm{O}^{\prime \prime} \mathrm{P}^{\prime \prime}}=\bar{l}_{2}^{\prime} R_{0}=-\frac{2 R_{0}}{\pi+1} .
\end{array}\right.
$$

Les conditions de stigmatisme étant analogues dans les deux traversées [6], cela signifie que les points stigmatiques images $\mathbf{C}^{\prime}$ et $\mathbf{P}^{\prime}$ de la première traversée coïncident avec les points stigmatiques objets de la deuxième, le sens de parcours de la trajectoire prin- cipale étant simplement inversé. Les points stigmatiques images $\mathrm{C}^{\prime \prime}$ et $\mathrm{P}^{\prime \prime}$ dans la deuxième traversée sont, puisque les trajectoires d'entrée et de sortie du «double prisme» sont alignées, les symétriques par rapport à l'axe de symétrie $\mathrm{C}^{\prime} \mathrm{O}^{\prime}$ des points stigmatiques objets dans la première traversée (Fig. 1 et 2).

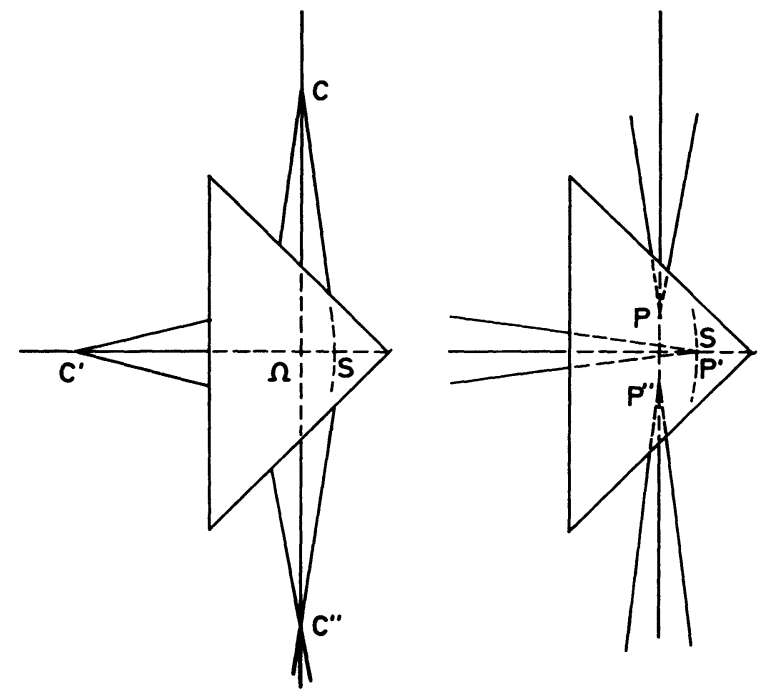

FIG. 2. - Points stigmatiques du système total lorsque le sommet $\mathrm{S}$ du miroir est au point stigmatique virtuel $\mathrm{P}^{\prime} ; \Omega$ : position de la focale achromatique.

I. 2 Chromatisme DU PRISMe (PREMIÈre traVerséE). - Lorsque la vitesse des particules incidentes varie, les rayons résultant de la dispersion de la trajectoire centrale $\mathrm{CO}$ après la première traversée semblent provenir d'un point $\Omega$ qui joue le rôle d'une focale achromatique $[3,6]$.

En effet, si la trajectoire moyenne est suivie par des

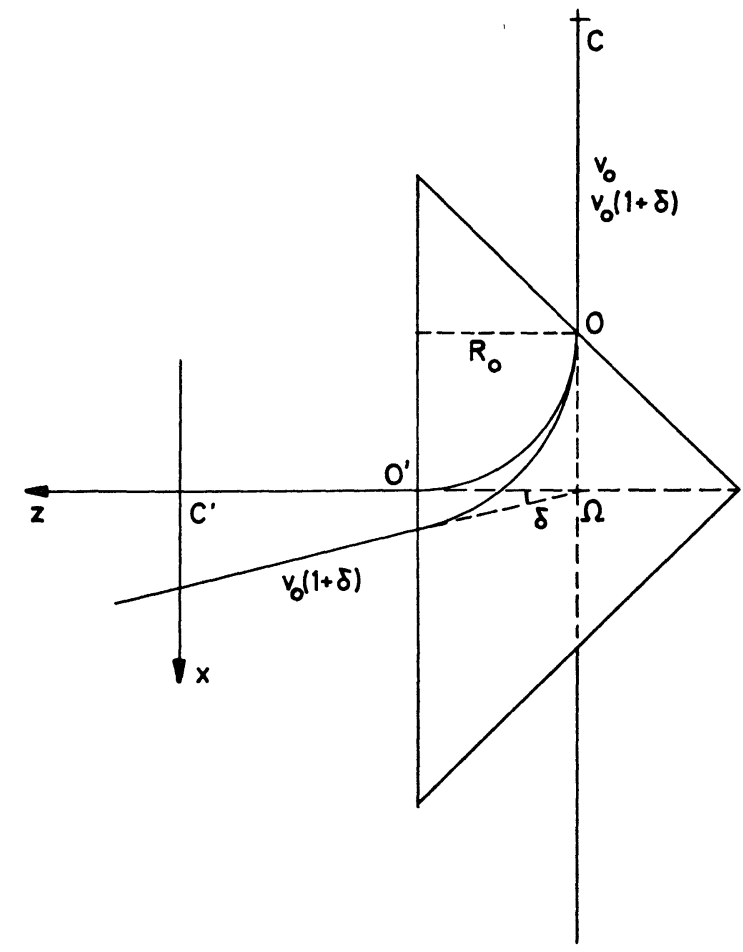

Fig. 3. - Focale achromatique $\Omega$ (première traversée du prisme) 
ions de vitesses $v_{0}$ et $v_{0}(1+\delta)$, où $\delta=\Delta v / v_{0}$, les ions de vitesse $v_{0}(1+\delta)$ emprunteront à la sortie une trajectoire de pente $\delta$ (Fig. 3), semblant provenir du point virtuel $\Omega$ sur l'axe, situé à la distance $R_{0}$ de la face de sortie du prisme : $\overline{\mathrm{O}^{\prime} \Omega}=-R_{0}$ [6].

La variation de vitesse $\delta$ étant directement reliée à la variation d'énergie $\Delta V / V\left(\delta=\frac{1}{2} \Delta V / V\right.$ au second ordre près), à la sortie du prisme, les trajectoires d'ions d'énergie comprise entre $V$ et $(V+\Delta V)$ qui ont suivi l'axe $\mathrm{CO}$, s'étalent en éventail dans le plan radial en s'appuyant sur une caustique tangente à l'axe de sortie en $\Omega$.

Toutes les trajectoires semblent donc, au second ordre près en $\delta$, provenir du point $\Omega$.

II. Position du miroir électrostatique. Position de l'objet. - Le prisme magnétique précédemment décrit doit nécessairement être associé à un système qui assurera le « retour » des particules après la première traversée. Ce système est ici un miroir électrostatique dont les éléments optiques, centre et sommet, peuvent être disposés de diverses manières par rapport au prisme, mais dont l'axe doit coïncider avec l'axe de symétrie. Il reste en plus à placer la pupille d'éclairement (ou "cross-over») du système (objectif de microscope, lentille à émission, etc...) qui donne l'image initiale qu'on désire filtrer. Comme le filtrage doit se faire au niveau d'un cross-over image réel, on placera la pupille d'éclairement au point $\mathrm{C}$ par exemple, et le sommet $\mathrm{S}$ ou le centre $\Gamma$ du miroir en $\mathrm{C}^{\prime}$; le filtrage s'effectuera alors, soit au niveau de $\mathrm{C}^{\prime \prime}$ après deux déflexions dans le premier cas, soit au niveau de $\mathrm{C}^{\prime}$ après une seule déflexion dans le second cas. Examinons ces deux cas :

II.1. - Dans le filtre de vitesses réalisé par Henry pour la microscopie électronique [5,6,7], le miroir est disposé de manière que son sommet $\mathrm{S}$ coïncide avec le point stigmatique réel $\mathrm{C}^{\prime}$ et son centre $\Gamma$ avec le point stigmatique virtuel $\mathbf{P}^{\prime}$ (miroir équivalent concave). On montre alors qu'il y a, pour l'ensemble double prisme-miroir un plan achromatique situé au niveau du point stigmatique virtuel $\mathrm{P}^{\prime \prime}$ si l'objectif du microscope forme une image en $\mathbf{P}$ (plan objet I pour le prisme). Le système prisme-miroir donne une image finale $\mathrm{I}^{\prime \prime}$ en $\mathrm{P}^{\prime \prime}$ qui est achromatique; l'image de la pupille initiale se trouve en $C^{\prime \prime}$ pour une valeur particulière de la quantité de mouvement des particules. A une autre valeur de la quantité de mouvement correspondent des trajectoires qui tournent différemment dans le prisme, et qui sont alors arrêtées par les lames de sélection placées au niveau de $\mathrm{C}^{\prime \prime}\left({ }^{1}\right)$.

II.2. - Dans le microanalyseur ionique auquel on s'intéresse plus particulièrement dans cette étude, le

(1) Il faut remarquer que, dans ce cas, la constitution même d'un miroir électrostatique dont le sommet doit se trouver en $\mathbf{C}^{\prime}$ et le centre en $\mathbf{P}^{\prime}$, fait que le sommet est un élément virtuel, et qu'il est par conséquent impossible d'y placer une fente de sélection. centre $\Gamma$ du miroir est en $\mathrm{C}^{\prime}$; le calcul montre alors que, quelle que soit la position du sommet, le point achromatique de la double traversée est en $\mathrm{C}^{\prime \prime}$. Il n'est donc plus possible de faire un filtrage après la traversée du système total ; le filtrage en quantité de mouvement s'effectue alors au niveau de $\mathrm{C}^{\prime}$ après la première traversée $\left({ }^{2}\right)$.

Nous étudions deux positions possibles du sommet $\mathrm{S}$ du miroir convexe : $\mathrm{S}$ peut se trouver, soit au point stigmatique $\mathrm{P}^{\prime}$, soit sur la focale achromatique $\Omega$.

II.2.a. - Lorsque $\mathrm{S}$ est en $\mathrm{P}^{\prime}$ (rayon du miroir $\left.\mathrm{r} R_{0}=\frac{\pi+2}{2} R_{0}\right)$, le système prisme-miroir est stigmatique quelle que soit la position du point objet sur l'axe [11], mais il n'est pas achromatique et il y aura de la dispersion sur l'image finale $\left({ }^{3}\right)$. Ceci résulte simplement du fait que, dans chaque section principale, la conjugaison des points objet et image se fait par une transformation homographique symétrique, et que, par suite, si les points doubles de chacune des deux transformations coïncident, les deux transformations sont identiques.

II.2.b. - Une autre disposition peut être obtenue en excitant la lentille-miroir de telle sorte que le sommet S coïncide avec la focale achromatique $\Omega$ dans la première traversée (rayon du miroir

$$
\left.\mathrm{r} R_{0}=R_{0}\left(t+\frac{3}{2}\right)\right) \text {. }
$$

Alors, les trajectoires moyennes des ions d'énergies différentes, qui semblent provenir, après la première traversée, du centre $\Omega$ du prisme, seront réfléchies symétriquement par rapport à l'axe $\mathrm{C}^{\prime} \mathrm{O}^{\prime}$ du miroir et subiront donc, dans la seconde traversée, une déviation symétrique de la première. Le système prisme-miroir est alors entièrement achromatique (Fig. 4). Il n'y a plus de dispersion au premier ordre sur l'image finale, quelle que soit sa position, donc quelle que soit la position de I.

Il est donc intéressant, dans ce cas, d'étudier quelle serait la meilleure position de l'objet, c'est-à-dire celle qui minimiserait les aberrations géométriques, principalement les aberrations de champ.

Comme dans cette disposition, le système dispersif n'est plus stigmatique pour des points objets de l'axe, il faut placer à la sortie un correcteur d'astigmatisme ramenant les deux focales l'une sur l'autre.

(2) Dans le cas II.2, il faut naturellement construire un miroir dont le centre se trouve effectivement hors du champ électrique si on veut $y$ placer une fente.

(3) On peut d'ailleurs noter que si, au lieu d'un objet ponctuel, on a un plan objet I perpendiculaire à l'axe $\mathrm{CO}$ en $\mathrm{P}$ par exemple, on obtient une image finale $\mathrm{I}^{\prime \prime}$ de dimensions égales à celles de l'objet, située dans le plan orthogonal à $\mathrm{O}^{\prime \prime} \mathrm{C}^{\prime \prime}$ en $\mathrm{P}^{\prime \prime}$; le grandissement $g$ du système prisme-miroir est égal à $1(-1$ pour les points images obtenus à partir de pinceaux du plan radial, +1 pour des pinceaux de la deuxième section principale). De même, l'image du point $C$ étant $C^{\prime}$, centre du miroir convexe, la pupille de sortie sera dans le plan perpendiculaire à l'axe en $C^{\prime \prime}$, avec le grandissement linéaire +1 pour la première section principale et -1 pour la seconde. 


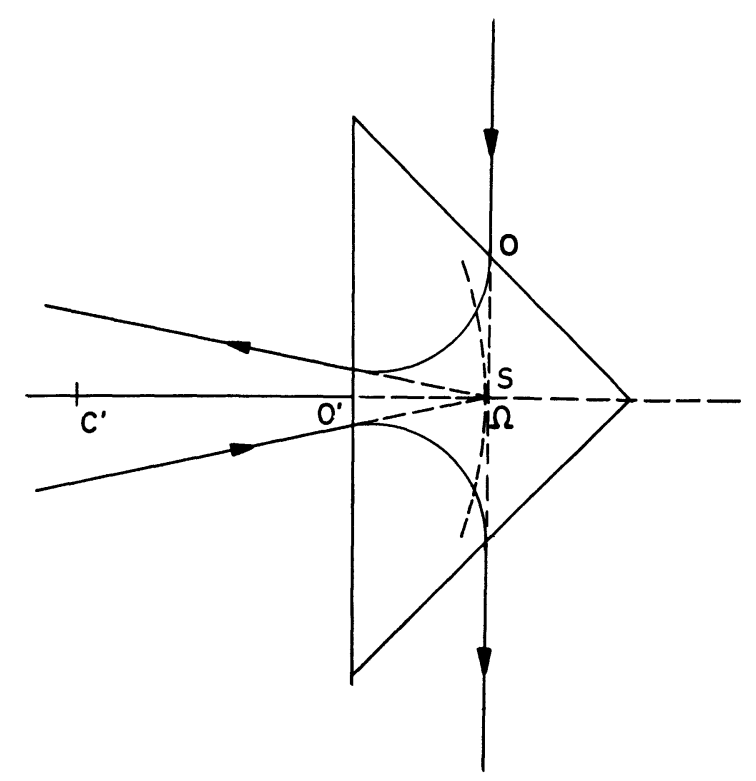

FIG. 4. - Système achromatique : le sommet $\mathrm{S}$ du miroir est en $\Omega$.

II. 3 REMARQUES. - Les positions des focales ont été déterminées par le calcul en négligeant l'existence des champs de fuite qui s'étendent en dehors des faces polaires. On sait que ceux-ci modifient ces positions de façon assez notable. Ceci est dû au fait que le faisceau commence à tourner avant d'aborder la limite réelle des pièces polaires. On peut en partie tenir compte des effets de cette rotation en calculant un « aimant théorique » dont les faces polaires seraient situées en avant des faces mécaniques [7, 12].

Néanmoins, nous étudierons les défauts du système dispersif en supposant que le champ magnétique est constant dans tout l'entrefer et qu'il s'annule brusquement à l'aplomb des faces polaires. Nous nous bornerons, dans cet article, à un calcul géométrique simple des trajectoires, en supposant, en outre, que le miroir ne présente aucune aberration, géométrique ou chromatique.

Comme le système dispersif n'est pas de révolution, les résultats trouvés à l'approximation de Gauss vont être modifiés par l'existence d'aberrations du second ordre (aberrations géométriques et chromatiques) dont les effets seront sensibles pour les positions des focales correspondant à un point objet pris dans le plan I, en dehors de l'axe.

Nous avons donc calculé les trajectoires en conservant tous les termes du second ordre par rapport aux coordonnées du point d'intersection d'une trajectoire incidente avec le plan de la pupille d'entrée.

Nous avons d'ailleurs retrouvé par le calcul analytique des trajectoires, les résultats de Wollnik [13] et de Enge [14] pour les termes d'aberrations correspondant au plan radial ; une différence apparaît cependant pour les termes hors du plan de symétrie donnés par Wollnik, qui est due à un désaccord dans le calcul de l'action des couches de passage. D'autre part, nous avons fait une approximation supplémentaire vis-à-vis des calculs de Enge en prenant une induction magnétique qui varie brusquement de zéro à $B_{0}$ à l'aplomb des faces polaires de l'aimant.

Par ailleurs, l'utilisation de la théorie de Cotte [9] mène à des résultats quelquefois différents de ceux du calcul géométrique. Ceci provient essentiellement du fait qu'il faudrait retoucher les calculs pour tenir compte de l'inclinaison des faces du prisme à l'ordre d'approximation qui nous intéresse, dans la détermination des fonctions de base qui figurent dans les matrices exprimant l'effet des couches de passage à l'entrée et à la sortie du prisme.

III. Aberrations du second ordre. - Nous étudions plus particulièrement ici le cas du miroir convexe dont le centre $\Gamma$ est en $\mathrm{C}^{\prime}$, et nous allons calculer les aberrations du second ordre pour les deux positions du sommet $\mathrm{S}$ indiquées plus haut.

Les calculs faits en appendice permettent d'obtenir les coordonnées de l'intersection avec le plan de la pupille de sortie $C^{\prime \prime}$ du prisme, d'une trajectoire caractérisée à l'entrée par ses coordonnées dans le plan de la pupille $\mathrm{C}$.

Dans le plan perpendiculaire à l'axe en $\mathrm{C}$, un rayon quelconque est défini par les coordonnées $x_{0}, y_{0}$ de son intersection avec le plan, par les pentes $p_{0}$ et $q_{0}$ de ses projections sur les deux sections principales, ainsi que par $\delta=\Delta v / v_{0}$.

Si $\lambda R_{0}$ est la distance séparant le plan du cross-over d'entrée $C$ du plan objet $I$, mesurée en rayons de courbure de la trajectoire principale dans le prisme (Fig. 5), et si $\alpha$ et $\beta$ sont les pentes des projections sur les deux sections principales (Fig. 5 et 6 ) d'une trajec-



FIG. 5. - Projection sur le plan radial d'un faisceau incident issu de la pupille d'entrée et convergeant au point $E$ (E se projette en A sur la trace du plan objet I). $\mathrm{CM}=\lambda R_{0}$ : distance pupille d'entrée - plan objet I.

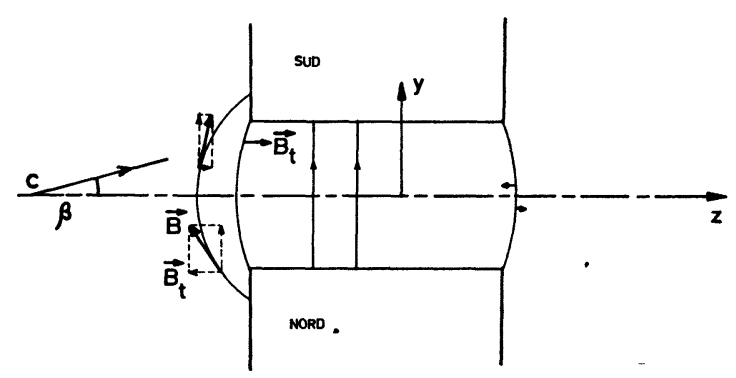

Fig. 6. $-\beta$ angle avec l'axe optique de la projection d'une trajectoire issue de $\mathrm{C}$ sur la deuxième section principale. $B_{\mathrm{t}}$ induction transversale agissant à la traversée des couches d'entrée et de sortie du prisme. 
toire issue du point $\mathrm{C}$ et rencontrant le plan $\mathrm{I}$ au point $\mathrm{E}$, intersection de la trajectoire définie par $\left(x_{0}, y_{0}, p_{0}, q_{0}\right)$ avec le même plan I, on a :

$$
p_{0}=\alpha-\frac{x_{0}}{\lambda R_{0}} \quad \text { et } \quad q_{0}=\beta-\frac{y_{0}}{\lambda R_{0}}
$$

$x_{0} / \lambda R_{0}$ et $y_{0} / \lambda R_{0}$ représentant les demi-ouvertures de pinceaux de trajectoires convergeant en $\mathrm{E}$ (le point $\mathrm{E}$ se projette en A sur le plan radial : Fig. 5).

Le miroir étant supposé parfait, nous pouvons étudier les aberrations dues au double prisme seul.

Les aberrations géométriques, qui dépendent de ces paramètres peuvent se classer en trois catégories [3] :

\section{Aberrations :}

- de distorsion : en $\alpha^{2}, \beta^{2}, \alpha \beta$; ces termes, qui n'affectent pas la résolution de l'appareil, dépendent de l'inclinaison des rayons joignant le centre de la pupille $\mathrm{C}$ aux points de l'objet.

- d'ouverture : en $x_{0}^{2}, y_{0}^{2}, x_{0} y_{0}$; ces termes dépendent des dimensions de la pupille d'entrée, donc de l'ouverture des pinceaux de trajectoires.

- de champ : en $x_{0} \alpha, y_{0} \alpha, x_{0} \beta, y_{0} \beta$; ces termes expriment le fait que les plans images ne sont pas forcément perpendiculaires à l'axe.

On peut également distinguer trois catégories d'aberrations chromatiques :

\section{Aberrations :}

- de champ : en $\alpha \delta, \beta \delta$.

- « mixte d'ouverture » : en $x_{0} \delta, y_{0} \delta$.

- terme en $\delta^{2}$.

Nous étudierons séparément chaque groupe d'aberrations, l'aberration totale qui entache un point de l'image étant formée par la réunion de tous les groupes; mais nous allons nous intéresser d'abord aux aberrations géométriques de champ et à leurs variations en fonction de la distance réduite $\lambda$ du plan objet I à la pupille $\mathrm{C}$.

III. 1 ABERRATIONS GÉOMÉTRIQUES DE CHAMP. Considérons tout d'abord un pinceau plat contenu dans le plan de symétrie qui s'appuie sur le diamètre de la pupille d'entrée $\mathrm{C}$ et qui irait converger en un point A du plan objet I (Fig. 5). L'axe CA de ce pinceau fait l'angle $\alpha$ avec CO. A la sortie du double prisme, ce pinceau diverge en semblant provenir du point virtuel $\mathrm{A}_{\mathrm{r}}^{\prime \prime}$, situé à l'intersection des prolongements des trajectoires rectilignes émergentes (Fig. 7).

L'intersection d'une trajectoire quelconque $\beta=0$ avec le plan du cross-over $C^{\prime \prime}$ est déterminée par $\left({ }^{4}\right)$ :

$$
\bar{x}_{5}=x_{0}-\mathcal{B}_{\mathrm{r}} x_{0} p_{0}
$$

(4) Les valeurs numériques des coefficients $\mathcal{A}, \mathfrak{B}$, $\mathcal{C}$ sont données dans l'Appendice I (relations I.10 et I.11) pour les deux positions du sommet $\mathbf{S}$ du miroir ; (indices $\mathbf{r}$ pour le plan radial, a pour la $2^{\mathrm{e}}$ section principale).

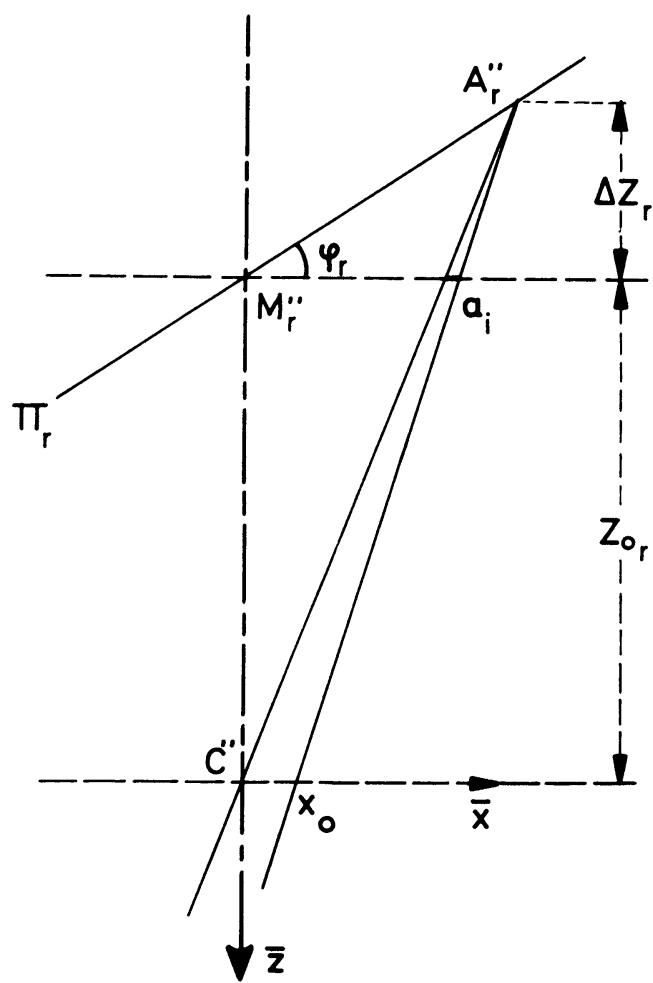

Fig. 7. - Pinceau image conjugué d'un pinceau objet plat du plan radial s'appuyant sur le rayon $x_{0}$ de la pupille d'entrée $C$ et convergeant en A dans le plan objet I, hors de l'axe $\mathrm{CO}$; $Z_{\text {or }}$ abscisse de $\mathbf{M}_{\mathbf{r}}^{\prime \prime}$, «image radiale » de $M$ sur l'axe. $\Delta Z_{\mathrm{r}}$ correction du premier ordre ; $a_{i}$ aberration de champ dans l'espace image $; \Pi_{\mathrm{r}}$ trace du plan lieu de la focale radiale.

$$
\vec{p}_{5}=\mathcal{A}_{\mathrm{r}} \frac{x_{0}}{R_{0}}+p_{0}+\mathcal{C}_{\mathrm{r}} \frac{x_{0} p_{0}}{R_{0}}+\mathcal{D}_{\mathrm{r}} p_{0}^{2}
$$

L'intersection de cette trajectoire avec le plan d'abscisse $Z_{\mathrm{r}}$ comptée sur l'axe $\mathrm{O}^{\prime \prime} \mathrm{C}^{\prime \prime}$ à partir de $\mathrm{C}^{\prime \prime}$ est donnée par $\bar{x}=\bar{p}_{5} Z_{\mathrm{r}}+\bar{x}_{5}$.

Pour qu'on puisse dire que $A_{r}^{\prime \prime}$ est l'image du point objet $A$, il faut que sa position soit indépendante du rayon du pinceau convergeant en $\mathrm{A}$; il faut donc que $\bar{x}$ soit indépendant de $x_{0}$, ouverture du pinceau objet, ce qu'on obtient pour une valeur de $Z_{\mathrm{r}}$ telle que :

$$
\begin{array}{r}
Z_{\mathrm{r}}=-\frac{\lambda R_{0}}{\mathcal{A}_{\mathrm{r}} \lambda-1}\left\{1-\alpha \mathfrak{B}_{\mathrm{r}} \frac{\mathcal{A}_{\mathrm{r}} \lambda-2+\mathcal{C}_{\mathrm{r}} \lambda / \mathscr{S}_{\mathrm{r}}}{\mathcal{A}_{\mathrm{r}} \lambda-1}\right\}= \\
=Z_{0 \mathrm{r}}+\Delta Z_{\mathrm{r}}
\end{array}
$$

La valeur correspondante de $\bar{x}$ est :

$$
\begin{aligned}
\bar{x}=Z_{\mathrm{r}} \alpha= & -\frac{\lambda R_{0} \alpha}{A_{\mathrm{r}} \lambda-1}- \\
& -\alpha^{2} \lambda R_{0} \frac{\mathscr{B}_{\mathrm{r}}\left(2-\mathcal{A}_{\mathrm{r}} \lambda-\mathcal{C}_{\mathrm{r}} \lambda / \mathscr{B}_{\mathrm{r}}\right)}{\left(\mathcal{A}_{\mathrm{r}} \lambda-1\right)^{2}}
\end{aligned}
$$

Pour $\alpha=0, \mathrm{~A}_{\mathrm{r}}^{\prime \prime}$ vient se confondre avec le point image $M_{r}^{\prime \prime}$ sur l'axe, dont la distance au cross-over de sortie $C^{\prime \prime}$ est :

$$
Z_{0_{\mathrm{r}}}=-\frac{\lambda R_{0}}{A_{\mathrm{r}} \lambda-1}
$$


Lorsque $\alpha$ n'est pas nul, le segment AM contenu dans le plan radial et perpendiculaire à l'axe, a pour image un segment de droite $A_{r}^{\prime \prime} M_{r}^{\prime \prime}$ qui n'est plus perpendiculaire à l'axe en $M_{r}^{\prime \prime}$, mais qui fait avec la normale à $\mathrm{O}^{\prime \prime} \mathrm{C}^{\prime \prime}($ axe $\bar{x})$, un angle $\varphi_{\mathrm{r}}$ (Fig. 7) tel que :

$$
\operatorname{tg} \varphi_{\mathrm{r}}=\frac{\Delta Z_{\mathrm{r}}}{\bar{x}}=\mathfrak{B}_{\mathrm{r}} \frac{2-\mathcal{A}_{\mathrm{r}} \lambda-\mathcal{C}_{\mathrm{r}} \lambda / \mathcal{B}_{\mathrm{r}}}{\mathcal{A}_{\mathrm{r}} \lambda-1}
$$

au premier ordre près en $\alpha$ (le terme qu'on néglige contribuerait à une "courbure de champ ").

Ceci est dû au fait qu'une trajectoire correspondant à un angle $\alpha$ positif parcourt dans l'entrefer une distance plus grande que la trajectoire centrale $(\alpha=0)$, et est donc soumise à une action convergente un peu plus forte, ce qui a pour effet de rendre le pinceau image issu de $A_{r}^{\prime \prime}$ moins divergent que celui issu de $\mathbf{M}_{\mathrm{r}}^{\prime \prime}$, donc d'éloigner davantage du cross-over le point $\mathrm{A}_{\mathrm{r}}^{\prime \prime}$. Pour un angle $\alpha$ négatif, l'action convergente est moins forte et le point image est alors plus proche de $\mathrm{C}^{\prime \prime}$ que $\mathrm{M}_{\mathrm{r}}^{\prime \prime}$. La courbure de champ d'un système optique gaussien est remplacée ici par un défaut d' 'inclinaison de champ" (terme du second ordre alors que la courbure de champ d'un système de révolution provient de termes du troisième ordre).

Pour des pinceaux plats contenus dans les surfaces cylindriques analogues à la seconde section principale, mais correspondant à un angle $\alpha$ non nul (que l'on pourra appeler «deuxièmes sections secondaires" en se référant à l'optique géométrique classique), nous pouvons examiner de la même manière, la convergence au troisième ordre près, des pinceaux images.

Chaque pinceau plat incident est caractérisé par son ouverture $y_{0}\left(x_{0}=0\right)$. A la sortie, une trajectoire rectiligne correspondant à un angle $\beta$ à l'entrée du système aura pour équation :

$$
\begin{array}{r}
\bar{y}=\bar{q}_{5} Z_{\mathrm{a}}+\bar{y}_{5}=Z_{\mathrm{a}}\left(-\mathcal{A}_{\mathrm{a}} \frac{y_{0}}{\mathrm{R}_{0}}-q_{0}-\mathscr{D}_{\mathrm{a}} p_{0} q_{0}\right)- \\
-y_{0}+\mathscr{B}_{\mathrm{a}} y_{0} p_{0} .
\end{array}
$$

La valeur de $Z_{\mathrm{a}}$ correspondant à la focalisation du pinceau image a la même expression en fonction de $\alpha$ que $Z_{\mathbf{r}}$ (relation (4)), si on remplace les coefficients $\mathcal{A}_{\mathrm{r}}, \mathfrak{B}_{\mathrm{r}}, \mathrm{C}_{\mathrm{r}}$ relatifs à la première section principale par $\mathcal{A}_{\mathrm{a}}, \mathfrak{B}_{\mathrm{a}}, \mathrm{C}_{\mathrm{a}}$ relatifs à la seconde section $\left({ }^{4}\right)$ :

$$
Z_{\mathrm{a}}=Z_{0 \mathrm{a}}+\Delta Z_{\mathrm{a}}
$$

où $\Delta Z_{\mathrm{a}}$ est linéaire en $\alpha . Z_{\mathrm{a}}$ est donc indépendant de $\beta$. Par suite, l'image d'un petit segment de droite EA $\mathrm{du}$ plan objet I, perpendiculaire au plan de symétrie en $\mathrm{A}$, est un segment de droite virtuel $\mathrm{E}_{\mathrm{a}}^{\prime \prime} \mathrm{A}_{\mathrm{a}}^{\prime \prime}$, également perpendiculaire au plan de symétrie.

Lorsque le point objet $\mathrm{E}$ balaie la surface du plan I, perpendiculaire à l'axe $\mathrm{CO}$ en $\mathrm{M}$, avec des pinceaux plats qui s'appuient sur le diamètre de la pupille d'entrée situé dans le plan de symétrie, l'image donnée par le système double prisme miroir est un plan perpen- diculaire au plan de symétrie dont la trace sur ce dernier est $M_{r}^{\prime \prime} A_{r}^{\prime \prime}$ (Fig. 7). Lorsque le faisceau est plein et que $\mathrm{E}$ décrit le plan $\mathrm{I}$, le pinceau émergent s'appuie sur deux focales dont les lieux géométriques sont deux plans $\Pi_{\mathrm{r}}$ et $\Pi_{\mathrm{a}}$, perpendiculaires au plan radial, mais inclinés sur l'axe optique, et dont les traces sur le plan radial font respectivement avec l'axe $\bar{x}$ orthogonal à $\mathrm{O}^{\prime \prime} \mathrm{C}^{\prime \prime}$, des angles $\varphi_{\mathrm{r}}$ et $\varphi_{\mathrm{a}}\left(\operatorname{tg} \varphi_{\mathrm{r}}\right.$ étant donnée par (7) et $\operatorname{tg} \varphi_{\mathrm{a}}$ étant déterminée de manière semblable par $\operatorname{tg} \varphi_{\mathrm{a}}=\Delta Z_{\mathrm{a}} / \bar{x}$ ) (Fig. 8).

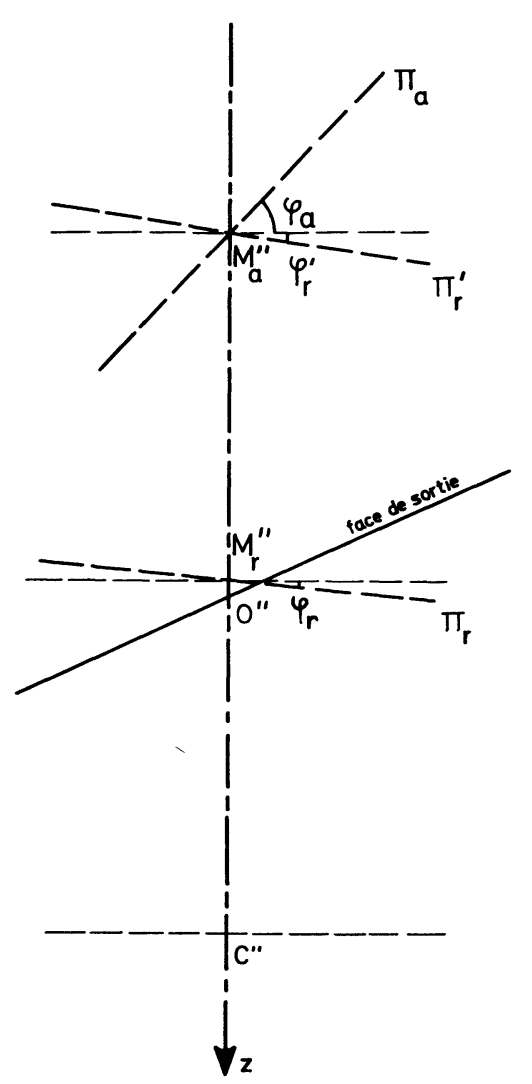

Fig. 8. - Traces des plans $\Pi_{\mathrm{r}}$ et $\Pi_{\mathrm{a}}$, lieux des focales, faisant les angles $\varphi_{\mathrm{r}}$ et $\varphi_{\mathrm{a}}$ avec la normale à l'axe z. $\Pi_{\mathrm{r}}^{\prime}$ image de $\Pi_{\mathrm{r}}$ donnée par le correcteur d'astigmatisme (angle $\left.\varphi_{\mathbf{r}}^{\prime}\right)$.

D'autre part, il est nécessaire, pour compenser l'astigmatisme sur l'axe, d'utiliser une lentille correctrice qui, de la focale radiale $\mathrm{M}_{\mathrm{r}}^{\prime \prime}$ (ou $\mathrm{P}_{\mathrm{r}}^{\prime \prime}$ dans le cas particulier où le point objet est en $\mathrm{P}$ ), donnera une image confondue avec la focale axiale [3]. Cette lentille transforme une trajectoire émergente $\left[\bar{p}_{5}, \bar{x}_{5}\right]$ en $\left[\bar{p}_{5}-k \bar{x}_{5}=\bar{p}, \bar{x}_{5}\right]$. On trouve encore un angle $\varphi_{\mathrm{r}}^{\prime}$ entre la trace du plan corrigé $\Pi_{\mathrm{r}}^{\prime}$ (Fig. 8) et l'axe $\bar{x}$.

Nous avons étudié, par un calcul sur machine, les variations des angles $\varphi_{\mathrm{r}}^{\prime}$ et $\varphi_{\mathrm{a}}$ avec $\lambda$, suivant que le sommet $\mathrm{S}$ du miroir est sur la focale achromatique $\boldsymbol{\Omega}$ ou sur le point stigmatique $\mathrm{P}^{\prime}$ (courbes Fig. 9).

Lorsque $\mathrm{S}$ est en $\Omega$, le point de rencontre d'une trajectoire corrigée $\left[\bar{x}_{5}, \bar{p}\right]$ avec le plan d'abscisse $Z_{\mathrm{r}}=Z_{\mathrm{a}}=Z_{0}$ a pour coordonnée 


$$
\begin{aligned}
\bar{x}=\bar{p} Z_{0}+x_{5} & =-\frac{\lambda R_{0}}{\mathcal{A}_{\mathrm{r}} \lambda-1}\left[\frac{x_{0}}{R_{0}}\left(\frac{\mathcal{A}_{\mathrm{r}} \lambda-1}{\lambda}\right)+\right. \\
+ & \left.\alpha+\frac{x_{0} \alpha}{R_{0}}\left(\frac{\mathcal{C}_{\mathrm{r}} \lambda-2 \mathcal{D}_{\mathrm{r}}}{\lambda}\right)\right]+x_{0}-\mathcal{B}_{\mathrm{r}} x_{0} \alpha= \\
= & -\frac{\lambda R_{0}}{\mathcal{A}_{\mathrm{r}} \lambda-1} \alpha+x_{0} \alpha \cdot \mathcal{B}_{\mathrm{r}} \frac{2-\mathcal{A}_{\mathrm{r}} \lambda-\mathcal{C}_{\mathrm{r}} \lambda / \mathcal{B}_{\mathrm{r}}}{\mathcal{A}_{\mathrm{r}} \lambda-1}
\end{aligned}
$$$$
\left(\operatorname{avec} 2 \mathfrak{D}_{\mathrm{r}}=\mathcal{B}_{\mathrm{r}}\right)
$$

ou

$$
\bar{x}=Z_{0} \alpha+x_{0} \alpha \cdot \operatorname{tg} \varphi_{\mathrm{r}}^{\prime}=Z_{0} \alpha+a_{i_{\mathrm{r}}}
$$

en posant :

$$
a_{i_{\mathrm{r}}}=x_{0} \alpha \cdot \operatorname{tg} \varphi_{\mathrm{r}}^{\prime} .
$$

L'aberration de champ dans le plan image est donc mesurée, en valeur absolue et en unités $x_{0} \alpha$ par $2 \operatorname{tg} \varphi_{\mathrm{r}}^{\prime}, a_{i_{\mathrm{r}}}$ donnant la distance algébrique du point aberrant au point d'intersection de la trajectoire passant par C" avec le plan d'abscisse $Z_{0}$ (Fig. 7). Les conclusions sont analogues pour l'autre section et conduisent à une aberration $a_{i_{\mathrm{a}}}$.

Comme les $a_{i}$ dépendent de la position du plan
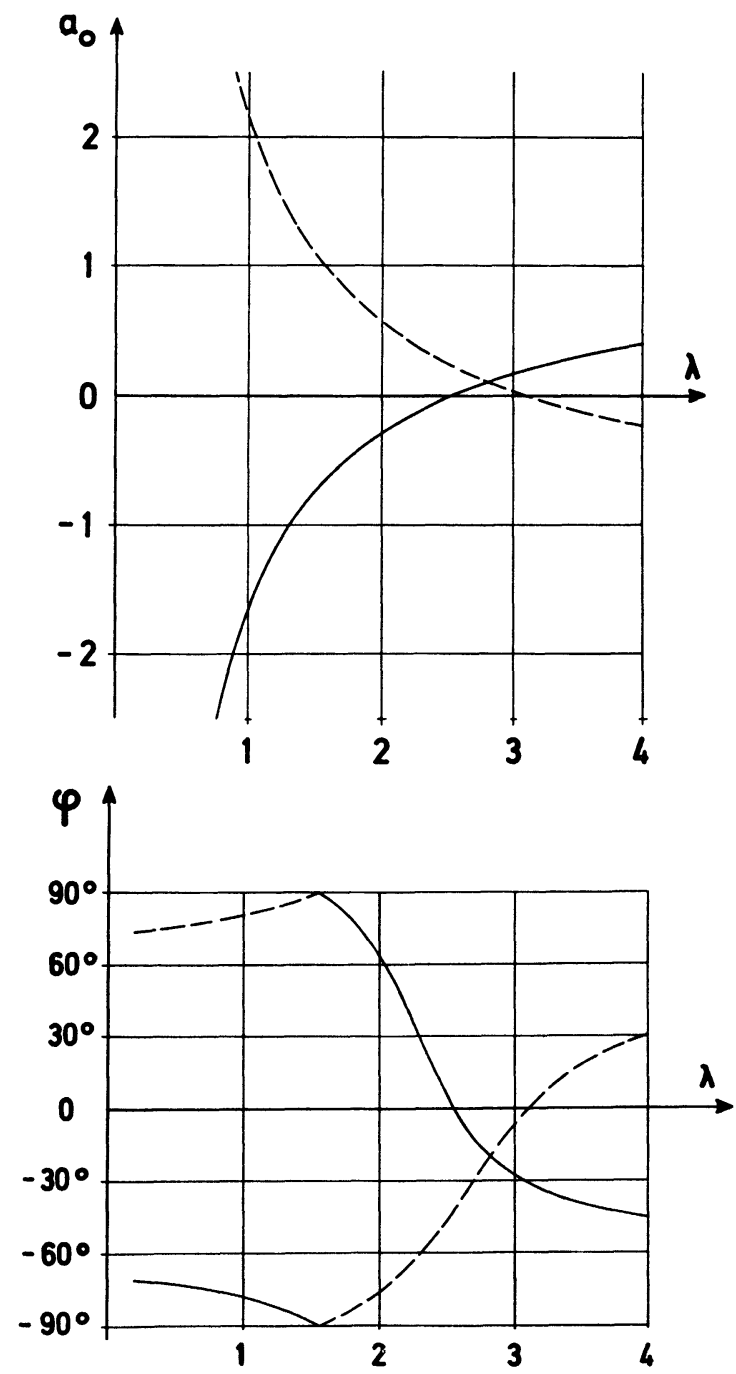

FIG. 9. - Astigmatisme de champ : $a_{0}$ aberration de champ rapportée au plan objet ; $\varphi$ angle de la trace du plan lieu de la focale, $\lambda$ distance du point objet sur l'axe au point $C$.

- courbes $a_{0}(\lambda)$ pour $S$ en $\Omega$ (a) et pour $\mathrm{S}$ en $\mathbf{P}^{\prime}(\mathrm{b})$ - courbes $\varphi(\lambda)$ pour $\mathrm{S}$ en $\boldsymbol{\Omega}$ (c) et pour $\mathrm{S}$ en $\mathrm{P}^{\prime}$ (d). Courbes en trait plein : focale radiale; en pointillés : focale axiale. image, donc du grandissement total $\mathrm{G}$ de l'appareil, y compris l'objectif placé devant le système dispersif, il est plus intéressant de se rapporter à l'objet (dont l'objectif donne l'image I). On obtient de la sorte directement l'influence de ces aberrations sur le pouvoir séparateur.

Si $a_{0}$ est l'aberration, comptée algébriquement, dans le champ objet de rayon $\rho$ (pour l'objectif $\rho=0,1 \mathrm{~mm}$ ), on a $G=a_{i} / a_{0}=\bar{x} / \rho$. Comme $\alpha \simeq \bar{x} / Z_{0}$

d'où

$$
a_{i}=x_{0} \cdot \operatorname{tg} \varphi \cdot \frac{\bar{x}}{Z_{0}}=a_{0} \cdot G,
$$

$$
a_{0}=\frac{x_{0} \rho}{Z_{0}} \operatorname{tg} \varphi
$$

Les longueurs des axes de la tache d'aberration (qui est elliptique comme nous allons le voir dans la suite) dans le champ objet, sont donc égales à $2\left|a_{0}\right|$ pour chaque section.

Nous avons tracé les courbes $a_{0}(\lambda)$ relatives aux deux focales, pour les deux positions du miroir et pour $\lambda$ variant de 0,2 à 4,0 (Fig. 9).
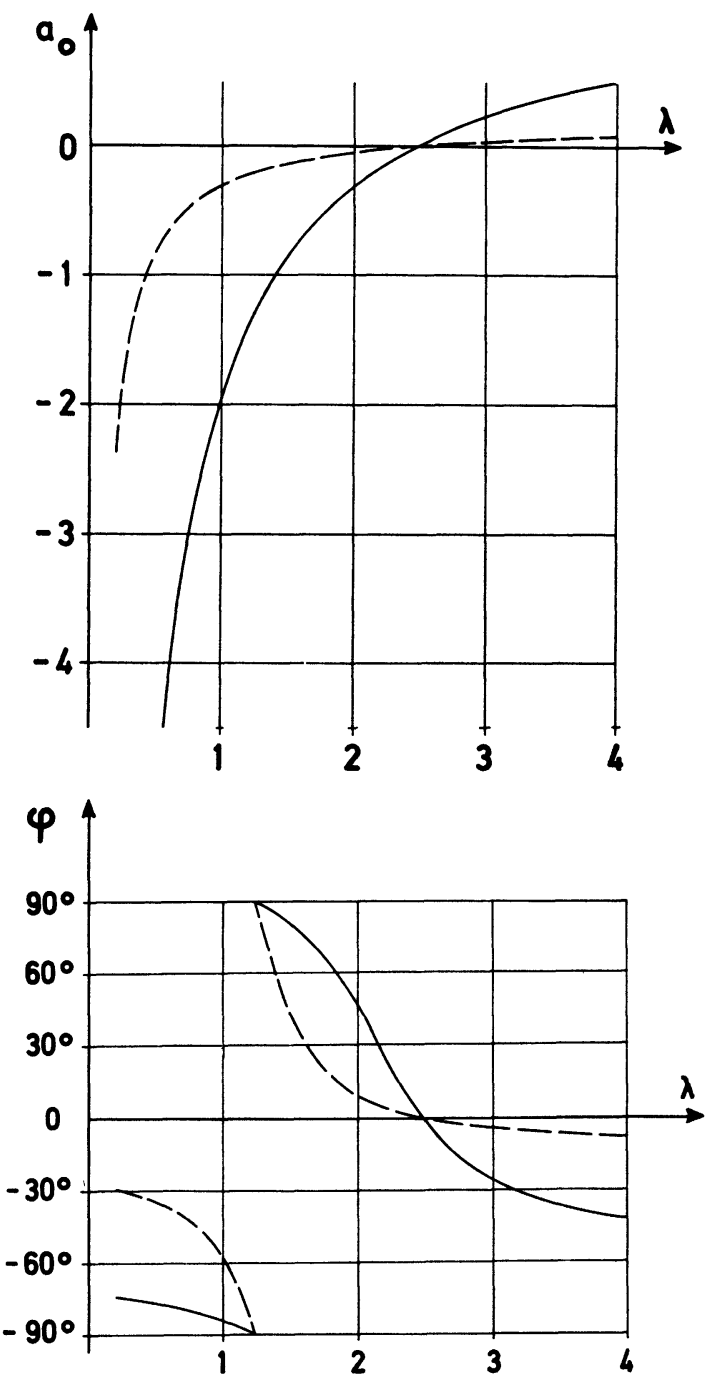
III.1.a. - Dans le cas où $\mathrm{S}$ est en $\mathrm{P}^{\prime}$ (stigmatisme), les fonctions $a_{0}(\lambda)$ s'annulent toutes deux lorsque le point objet sur l'axe est confondu avec $\mathrm{P}$, point stigmatique objet virtuel $(\lambda=2,483)$. Les courbes $\varphi(\lambda)$ qui traduisent la variation de la position des plans $\Pi$, lieux des focales, montrent que, lorsque $\lambda$ croît à partir de la position du foyer objet du système total ( $\lambda \simeq 1,24$ : les plans $\Pi$ n'ont, pour cette valeur, plus guère de signification), les traces sur le plan radial des plans $\Pi$ tendent à se rapprocher de la normale à $\mathrm{O}^{\prime \prime} \mathrm{C}^{\prime \prime}$, le redressement étant plus rapide pour $\Pi_{\mathrm{a}}$ que pour $\Pi_{\mathrm{r}}$. Les deux plans passent simultanément par la position perpendiculaire à $\mathrm{O}^{\prime \prime} \mathrm{C}^{\prime \prime}$ lorsque $\lambda=2,483$, puis ils se séparent de nouveau avec une rotation plus rapide pour $\Pi_{\mathrm{r}}$ que pour $\Pi_{\mathrm{a}}$ qui reste très voisin de la position perpendiculaire à l'axe. Il peut donc apparaître un certain astigmatisme de champ.

Mais lorsque l'objet est une petite surface plane, virtuelle, perpendiculaire à l'axe en $\mathrm{P}$, son image est une petite surface plane, virtuelle, également perpendiculaire à l'axe, et ceci, quel que soit le pinceau de trajectoires considéré. De plus, le grandissement $g$ du système prisme-miroir est égal à 1 comme nous l'avons signalé précédemment. Le système est donc aplanétique, à la courbure de champ près ; le pouvoir séparateur ne sera pas limité par les aberrations de champ du second ordre.

III.1.b. - Lorsque $\mathrm{S}$ est en $\Omega$ (avec le correcteur d'astigmatisme), les fonctions $a_{0}(\lambda)$ varient en sens inverse l'une de l'autre et prennent la même valeur, non nulle, pour $\lambda=2,82$ (alors $Z_{0}=-3,44 R_{0}$ et $g$ est supérieur à 1$)$. Les traces des plans $\Pi$, situées initialement (pour $\lambda$ voisin de 1,5 ) de part et d'autre de la normale à $\mathrm{O}^{\prime \prime} \mathrm{C}^{\prime \prime}$, se rapprochent l'une de l'autre quand $\lambda$ augmente, et les plans viennent se confondre pour $\lambda=2,82$. Leurs traces font alors un angle de $-1^{\circ}$ avec l'axe $\bar{x}$. Puis, lorsque $\lambda$ dépasse la valeur 2,82 , les plans s'écartent l'un de l'autre. Donc, quel que soit $\lambda$ autre que 2,82 , il y aura de l'astigmatisme pour un point pris hors de l'axe.

Effets de bord. - Si l'on se ramène, pour $\lambda$ quelconque, au plan perpendiculaire à l'axe en $\mathbf{M}_{\mathrm{a}}^{\prime \prime}$, l'intersection d'une trajectoire quelconque aura pour coordonnées : $\bar{x}_{Z}=Z_{0} \alpha+\Delta x$ et $\bar{y}_{Z}=-Z_{0} \beta-\Delta y$ où $Z_{0}$ est l'abscisse de $\mathrm{M}_{\mathrm{a}}^{\prime \prime} . \Delta x$ et $\Delta y$ contiennent ainsi des termes en $x_{0} \alpha, y_{0} \beta$ d'une part, en $y_{0} \alpha, x_{0} \beta$ d'autre part. Lorsque $x_{0}=y_{0}=0, \alpha=0$ et $\beta \neq 0$, la trajectoire ne reste pas dans la deuxième section principale et vient donner un terme en $\beta^{2}$ au niveau de $C^{\prime}$. Au passage des couches d'entrée et de sortie, l'ensemble du pinceau conique issu de la pupille d'entrée $C$ de de rayon $r_{0}$ et convergeant au point objet $E$, reçoit une impulsion qui rabat les trajectoires vers le plan radial (effet de focalisation), mais qui a aussi pour effet de le faire sortir de la deuxième section correspondante (cf. dans l'appendice I, le calcul de $\mathrm{p}_{2}$ en fonction de $p_{1}$ ). Cet effet est responsable de la pré- sence des termes en $x_{0} \beta$ et $y_{0} \beta$ qui traduisent ainsi l'existence d'un «effet de bord" [3].

Le point image gaussien $E_{a}^{\prime \prime}$ (Fig. 10), de coordonnées $Z_{0} \alpha,-Z_{0} \beta$, est entouré d'une figure d'aberration dont l'aspect est donné par les valeurs de $\Delta x$ et

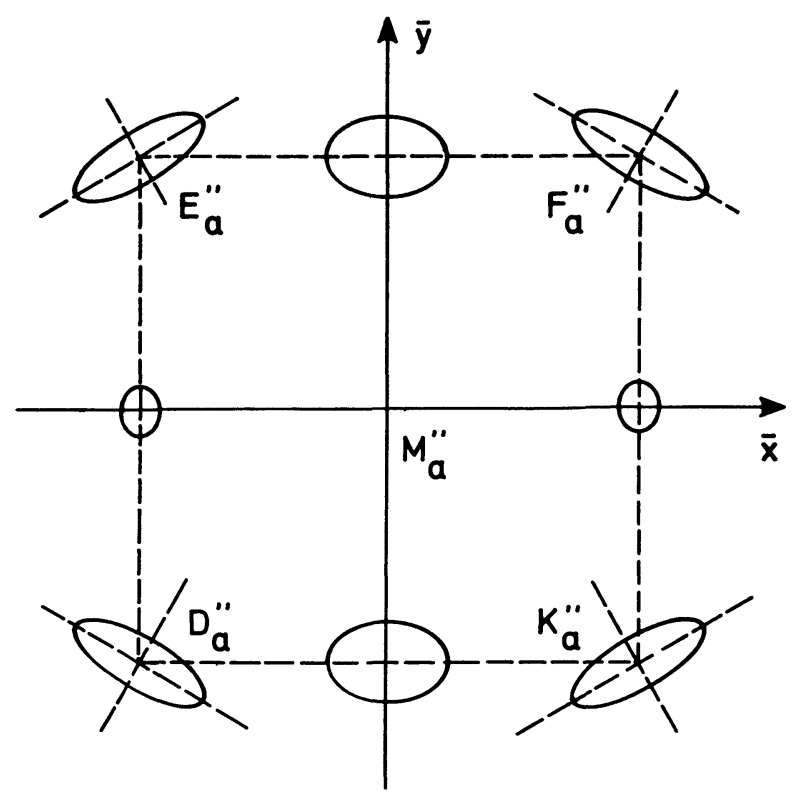

FIG. 10. - Aspect des taches d'aberration de champ dans un « plan image » d'abscisse $\mathbf{Z}$ comptée à partir de $C^{\prime \prime}$.

$\Delta y$. Nous pouvons étudier cette figure en ne considérant que les trajectoires qui s'appuient, au départ, sur le contour du cross-over situé en $\mathrm{C}$; on posera

$$
x_{0}=r_{0} \cos \psi \text { et } y_{0}=r_{0} \sin \psi
$$

avec $\psi$ variant de 0 à $2 \pi$. Les termes de l'aberration de champ forment un système linéaire en $\sin \psi$ et $\cos \psi$ d'où l'on peut éliminer $\psi$ pour obtenir l'équation du contour de la tache d'aberration.

C'est une ellipse centrée sur $E_{a}^{\prime \prime}$, dont les axes principaux font, avec les axes $\bar{x}, \bar{y}$ du plan perpendiculaire à l'axe en $\mathrm{M}_{a}^{\prime \prime}$, un angle $\omega$ dépendant de $\alpha$ et $\beta$, et variable avec $\lambda$. L'étude de la variation de la longueur des axes de la tache d'aberration avec la position de l'objet, ainsi que l'étude de $\omega$, montrent que, lorsque $\lambda$ varie, l'ellipse se déforme d'une manière très prononcée ; en particulier pour $2,4 \lesssim \lambda \lesssim 2,9$, on observe une permutation du grand et du petit axe de l'ellipse. Dans le cas où $\lambda=2,82$, la tache d'aberration entourant les points du plan radial $(\beta=0)$ est circulaire (puisque les valeurs de $a_{0}$ sont égales pour les deux sections principales), tandis que pour les points de l'axe $\bar{y}(\alpha=0)$, c'est une ellipse dont le grand axe est perpendiculaire à l'axe $\bar{y}$. Pour $\alpha=\beta$, les axes de l'ellipse font un angle $\omega$ d'environ $40^{\circ}$ avec les axes $\bar{x}, \bar{y}$.

On peut obtenir une valeur de $\lambda$ pour laquelle la tache entourant les points définis par $\alpha=0$ devient également un cercle, mais il est impossible de réduire cette tache à un point. 
Nous pouvons indiquer un ordre de grandeur de la tache d'aberration affectant chaque point de l'image gaussienne. Pour $\rho=0,1 \mathrm{~mm}$ et $r_{0}=0,2 \mathrm{~mm}$ (rayon du diaphragme de contraste), les dimensions de l'ellipse, rapportée à l'objet réel placé devant l'objectif, pour $R_{0}=100 \mathrm{~mm}$ et $\lambda=2,82$, sont de $0,04 \mu$ hors tout pour les points du plan radial; pour les points tels que $\alpha=\beta$, l'ellipse est inscrite dans un rectangle dont la longueur, suivant $\bar{x}$, est de $0,12 \mu$, et la largeur, suivant $\bar{y}$, de $0,09 \mu$.

III.2 AUTRES ABERRATIONS GÉOMÉTRIQUES. III.2.1 Distorsion. - Supposons que le contour du champ objet dans le plan I soit un carré DEFK centré sur l'axe principal du système, de côtés parallèles aux axes $x$ et $y$. Un rayon issu du point $C$ s'appuie sur ce contour. On a $x_{0}=y_{0}=0$ et $\delta=0$. La déformation du carré se traduit dans l'espace image par la présence des termes en $\alpha^{2}, \beta^{2}$, et $\alpha \beta$.

Dans le plan de la pupille de sortie du double prisme, $\bar{x}_{5}=0$ et $\bar{y}_{5}=0$ (Appendice I) quelle que soit la position du sommet $\mathrm{S}$ du miroir convexe. Il n'y a donc pas de distorsion au cross-over $C^{\prime \prime}$. Par suite, l'image de la pupille d'entrée circulaire de rayon $r_{0}$, est un cercle centré sur $C^{\prime \prime}$ et de rayon $r_{0}$. La double traversée du prisme a compensé exactement les aberrations de ce type sur le cross-over.

Néanmoins, comme les pentes $\bar{p}_{5}$ et $\bar{q}_{5}$ des trajectoires à la sortie contiennent des termes de distorsion, la figure obtenue en coupant par un plan quelconque perpendiculaire à l'axe $\mathrm{O}^{\prime \prime} \mathrm{C}^{\prime \prime}$, le faisceau image correspondant au faisceau issu de $\mathrm{C}$ et s'appuyant sur le carré DEFK, présente une certaine déformation; mais quelle que soit la position du plan de coupe, et quelle que soit la valeur de $\lambda$, la figure est déformée de la même manière puisque toutes les coupes perpendiculaires à $\mathrm{O}^{\prime \prime} \mathrm{C}^{\prime \prime}$ sont homothétiques dans une homo- thétie de centre $C^{\prime \prime}$. Il suffit donc d'étudier la coupe par un plan d'abscisse $Z_{0}$ comptée à partir de $C^{\prime \prime}$.

Cependant, l'aspect de la figure est différent suivant la position du sommet du miroir.

III.2.1.a. - Si $\mathrm{S}$ est en $\mathrm{P}^{\prime}$, l'intersection d'une trajectoire passant par $\mathrm{C}^{\prime \prime}$ qui correspond à un rayon issu de $\mathrm{C}$ et qui rencontre le plan $\mathrm{I}$ en un point du contour DEFK a pour coordonnées $\bar{x}=\bar{p}_{5} Z_{0}$ et $\bar{y}=\vec{q}_{5} Z_{0}$ :

$$
\begin{aligned}
& \bar{x}=Z_{0}\left(\alpha+0,82 \alpha^{2}-0,16 \beta^{2}\right) \\
& \bar{y}=-Z_{0}(\beta+0,26 \alpha \beta) .
\end{aligned}
$$

On obtient ainsi les transformées des côtés du carré. La figure est formée de quatre arcs de paraboles symétriques par rapport au plan radial. Les transformées des segments ED et $F K$ sont des $\operatorname{arcs} E^{\prime \prime} D^{\prime \prime}$ et $\mathrm{F}^{\prime \prime} \mathrm{K}^{\prime \prime}$ dont la concavité est tournée vers les $\bar{x}$ positifs pour $Z_{0}$ négatif, alors que les arcs $E^{\prime \prime} F^{\prime \prime}$ et $\mathrm{K}^{\prime \prime} \mathrm{D}^{\prime \prime}$ ont leur concavité tournée vers les $\bar{x}$ négatifs (pour $Z_{0}$ négatif) (Fig. 11a). Le sommet de la parabole portant l'arc $\mathrm{E}^{\prime \prime} \mathrm{F}^{\prime \prime}$ a une cote $\bar{y}$ inférieure à la cote des points $\mathrm{E}^{\prime \prime}$ et $\mathrm{F}^{\prime \prime}$.

III.2.1.b. - Si $\mathrm{S}$ est en $\Omega$, les coordonnées de l'intersection $\mathrm{du}$ rayon sont:

$$
\begin{aligned}
& \bar{x}=Z_{0}\left(\alpha+0,68 \alpha^{2}-0,14 \beta^{2}\right) \\
& \bar{y}=-Z_{0}(\beta-1,61 \alpha \beta)
\end{aligned}
$$

( $\bar{x}$ est le même dans ce cas, avec ou sans correcteur d'astigmatisme). La figure obtenue est encore constituée de quatre arcs de paraboles se coupant aux points $\mathrm{D}^{\prime \prime} \mathrm{E}^{\prime \prime} \mathrm{F}^{\prime \prime} \mathrm{K}^{\prime \prime}$ avec les concavités disposées de la même manière que dans le cas précédent (toujours pour $Z_{0}$ négatif), mais le sommet de la parabole $E^{\prime \prime} F^{\prime \prime}$ a, cette
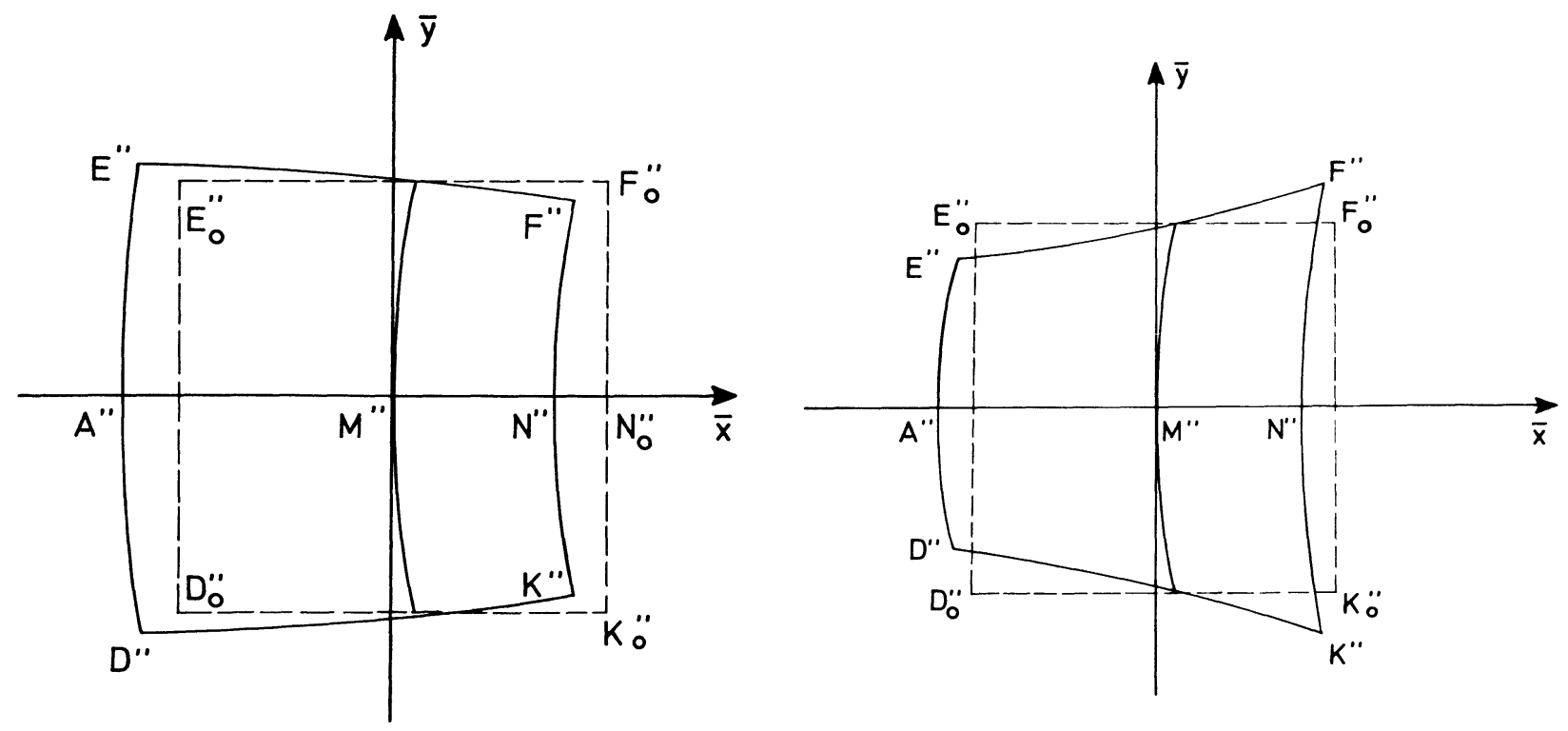

FIG. 11. - Distorsion :

- $a$ ) aspect de l'image lorsque $\mathrm{S}$ est en $\mathrm{P}^{\prime}$;

$-b$ ) aspect de l'image lorsque $\mathrm{S}$ est en $\Omega$. 
fois, une cote $\bar{y}$ supérieure à celle des points $\mathrm{E}^{\prime \prime}$ et $\mathrm{F}^{\prime \prime}$, ce qui donne l'aspect représenté figure $11 b$.

Lorsque $Z_{0}$ est positif, la figure $\mathrm{D}^{\prime \prime} \mathrm{E}^{\prime \prime} \mathrm{F}^{\prime \prime} \mathrm{K}^{\prime \prime}$ est symétrique de celle obtenue pour $Z_{0}$ négatif, par rapport au centre de la figure.

Nous pouvons donner un ordre de grandeur du taux de distorsion introduit sur l'image. Si on définit, le long de l'axe $\bar{x}$, un taux de distorsion $\tau_{x}$ par

$$
\tau_{x}=2 \frac{A^{\prime \prime} A_{0}^{\prime \prime}}{A_{0}^{\prime \prime} N_{0}^{\prime \prime}}
$$

(les indices 0 caractérisant l'image gaussienne), et, parallèlement à l'axe $\bar{y}$, un taux $\tau_{y}=2 \frac{E_{y}^{\prime \prime} E_{0}^{\prime \prime}}{D_{0}^{\prime \prime} E_{0}^{\prime \prime}}$ (en désignant par $\mathrm{E}_{y}^{\prime \prime}$ la projection de $\mathrm{E}^{\prime \prime}$ sur $\mathrm{E}_{0}^{\prime \prime} \mathrm{D}_{0}^{\prime \prime}$ ), on obtient, pour un angle $\alpha$ de $10^{-2}$ radian :

$\begin{array}{ccc}\text { Miroir } & \frac{\tau_{x}}{-} & \frac{\tau_{y}}{1,61} \alpha \\ \operatorname{sur} \Omega & 0,68 \alpha & \simeq 1,6 \% \\ \text { sur } \mathrm{P}^{\prime} & 0,7 \% & 0,26 \alpha \\ & \simeq 0,82 \% & \simeq 0,26 \%\end{array}$

Le taux de distorsion est donc de l'ordre de $1-1,5$ pour cent. L'image donnée par le prisme est déformée, mais, en principe, la distorsion n'influe pas sur la limite de résolution de l'appareil pour des particules monocinétiques.

III.2.2 Aberrations d'ouverture. - Elles proviennent du fait que la pupille d'entrée du système n'est pas de dimension infiniment petite, mais est caractérisée par un rayon $r_{0}$ petit. Ces aberrations n'influent que sur les points voisins du centre de l'image, car sur les bords du champ observé, elles sont négligeables devant les défauts de champ.

Nous pouvons cependant indiquer, dans le cas où $\mathrm{S}$ est en $\Omega$ et où $\lambda=2,82$, la forme de la tache d'aberration d'ouverture entourant chaque point du plan image I'. C'est une ellipse d'axes parallèles aux axes $\bar{x}, \bar{y}$, de demi-grand axe le long de $\bar{y} 0,80 r_{0}^{2} / R_{0}$ et de demi-petit axe suivant $\bar{x} 0,22 r_{0}^{2} / R_{0}$, ce qui donne avec les valeurs numériques déjà utilisées précédemment, une hauteur totale de $0,64 \mu$ en $\bar{y}$ pour une largeur de $0,18 \mu$ (Fig. 12). Ramenée au champ objet de rayon $\rho=0,1 \mathrm{~mm}$, l'ellipse a une dimension de $0,03 \mu$ le long de $y$ et de $0,008 \mu$ le long de $x$, ce qui est petit devant l'aberration de champ qui reste prépondérante sur les bords de l'objet.

Lorsque $\mathrm{S}$ est en $\mathrm{P}^{\prime}$, pour $\lambda=2,483$, on observe également une aberration d'ouverture, mais les dimensions de l'ellipse dans le plan image sont un peu plus faibles $\left(0,71 r_{0}^{2} / R_{0}\right.$ le long de $\bar{y}$ et $0,19 r_{0}^{2} / R_{0}$ le long de $\left.\bar{x}\right)$. Dans le champ objet, les axes de l'ellipse sont de $0,03 \mu$ en $y$ et $0,009 \mu$ en $x$.

III. 3 AberRations CHROMATIQUES. - On suppose maintenant que $\delta$ n'est pas nul. Les calculs faits en



Fig. 12. - Aberration d'ouverture.

appendice montrent qu'il apparaît des termes en $\alpha \delta$, $\beta \delta, x_{0} \delta, y_{0} \delta$ ainsi qu'en $\delta^{2}$, qui sont responsables des aberrations chromatiques.

Les coordonnées de l'intersection d'une trajectoire quelconque avec le plan image $I^{\prime \prime}$ d'abscisse $Z_{0}$, sont données, dans le cas du miroir convexe, par $\left({ }^{5}\right)$ :

$$
\begin{aligned}
\bar{x}_{Z} & =Z_{0} \alpha+\mathcal{A}_{1} Z_{0} \delta+x_{0} \delta\left[\mathscr{S}_{x}+\frac{\mathcal{C}_{x}}{\lambda}+\frac{\mathfrak{D}_{x} \lambda+\mathcal{E}_{x}}{1-\mathcal{A}_{r} \lambda}\right]- \\
& -R_{0} \alpha \delta\left[\mathcal{C}_{x}+\frac{\mathcal{E}_{x} \lambda}{1-\mathcal{A}_{r} \lambda}\right]+R_{0} \delta^{2}\left[\mathcal{A}_{\delta}+\mathfrak{B}_{\delta} \frac{\lambda}{1-\mathcal{A}_{r} \lambda}\right]
\end{aligned}
$$

$$
\begin{array}{r}
\bar{y}_{Z}=-Z_{0} \beta-y_{0} \delta\left[\mathscr{B}_{y}+\frac{\mathcal{C}_{y}}{\lambda}+\frac{\mathcal{D}_{y} \lambda+\mathcal{E}_{y}}{1-\mathcal{A}_{r} \lambda}\right]- \\
-R_{0} \beta \delta\left[\mathcal{C}_{y}+\frac{\mathcal{E}_{y} \lambda}{1-\mathcal{A}_{r} \lambda}\right] .
\end{array}
$$

Quelle que soit la position du sommet du miroir, l'expression de $\bar{x}_{5}$, abscisse de l'intersection d'une trajectoire avec le plan de la pupille de sortie $C^{\prime \prime}$, ne contient pas de terme du premier ordre en $\delta$. En conséquence, le système ne possède pas de «plan achromatique » à la différence des cas du prisme symétrique [3] et du double prisme associé à un miroir concave [6].

III.3.1 Lorsque $\alpha=\beta=0$, c'est-à-dire lorsqu'on considère un pinceau plein qui s'appuie sur le contour

(5) Les valeurs numériques des coefficients sont données dans l'appendice I (relations $\mathrm{I}-10, \mathrm{I}-11$ et $\mathrm{I}-12$ ). Les $\mathfrak{B}$ et $\mathcal{C}$ représentent les coefficients des aberrations chromatiques dans les expressions de $\bar{x}_{5}$ et $\bar{y}_{5}$; les $\mathcal{D}$ et $\mathcal{E}$ sont les coefficients de ces mêmes termes dans $\bar{p}_{5}$ et $\bar{q}_{5}$.

$\mathcal{A}_{\delta}$ et $\mathfrak{乃}_{\delta}$ sont les coefficients des termes en $\delta^{2}$ 
circulaire de la pupille d'entrée et qui converge en un point objet $\mathbf{M}$ de l'axe, la figure d'aberration chromatique due aux termes en $x_{0} \delta, y_{0} \delta$ est une ellipse dont le grand axe est en général parallèle à l'axe $\bar{y}$, donc au vecteur induction $\mathbf{B}_{0}$.

III.3.1. $a$ Quand le sommet du miroir est sur la focale achromatique $\Omega$, les calculs sur machine montrent que l'influence d'une variation de $\lambda$ entre 0 et 2,0 est très grande sur la longueur des axes $a_{x}$ et $a_{y}$ de la tache d'aberration. Par contre, $a_{x}$ et $a_{y}$ restent à peu près constants lorsque $\lambda$ varie de 2,0 à 4,$0 ; a_{x}$ est alors très petit, mais $a_{y}$, axe parallèle au vecteur $\mathbf{B}_{0}$, conserve une longueur notable.

On peut néanmoins réduire la tache à un «cercle de moindre diffusion " pour $\lambda$ voisin de 2,0 , mais l'objet se situe alors au voisinage du foyer objet du système, et pour une faible variation de $\lambda$, la tache se déforme extrêmement vite. D'autre part, dans ce cas, les défauts géométriques d'astigmatisme sont très importants.

Lorsque le point objet $\mathrm{M}$ est à la distance $\lambda=2,82$ de $\mathrm{C}$, pour un diaphragme de contraste de rayon $r_{0}=0,2 \mathrm{~mm}$ et une variation relative des vitesses $\delta=1 / 800$ fixée par les lames de sélection placées au niveau de $C^{\prime}$, les dimensions de l'ellipse dans le champ image sont $a_{x}=0,165 r_{0} \delta \simeq 0,041 \mu$ et $a_{y}=1,5$ $r_{0} \delta \simeq 0,38 \mu$. Compte tenu du grandissement total $G=23,5$, les dimensions de la tache dans le champ objet de rayon $\rho=100 \mu$ sont $a_{x_{0}} \simeq 0,002 \mu$. et $a_{y_{0}} \simeq 0,016 \mu$. Les défauts géométriques restent donc prépondérants devant les aberrations chromatiques.

III.3.1.b Lorsque le sommet $\mathrm{S}$ du miroir est au point stigmatique $\mathbf{P}^{\prime}$, les résultats sont similaires, la seule différence étant que, lorsque $\lambda$ croît de 2,0 à 4,0 , la longueur de l'axe $a_{y}$ marque une légère décroissance. Avec les mêmes données numériques que ci-dessus et pour $\lambda=2,483$, on a $a_{x} \simeq 0,21 r_{0} \delta=0,05 \mu$ et $a_{y} \simeq 1,3 r_{0} \delta=0,32 \mu$. Avec un grandissement $\mathrm{G}$ de 17, l'ellipse rapportée au champ objet a pour axes $a_{x_{0}} \simeq 3 \times 10^{-3} \mu$ et $a_{y_{0}} \simeq 0,02 \mu$.

III.3.2 Pour un point objet E quelconque du plan I, il se superpose, à l'aberration "mixte d'ouverture " qui vient d'être calculée, une aberration chromatique de champ provenant des termes en $\alpha \delta, \beta \delta$. Pour le point $\mathrm{E}$ et son image gaussienne $\mathrm{E}_{0}^{\prime \prime}, \alpha$ et $\beta$ sont fixes, mais comme $\lambda$ varie dans une certaine bande d'énergie, le défaut en $\alpha \delta, \beta \delta$ se traduit par l'existence d'un petit segment $E_{0}^{\prime \prime} E^{\prime \prime}$ de longueur proportionnelle à $\lambda$. Les termes en $\alpha \delta, \beta \delta$ varient assez fortement avec $\lambda$ (fonctions homographiques de $\lambda$ ).

Les coordonnées de $\mathrm{E}_{0}^{\prime \prime}$ sont $\mathrm{Z}_{0} \alpha,-\mathrm{Z}_{0} \beta$; celles de $\mathrm{E}^{\prime \prime}$ sont $\bar{x}_{z}=Z_{0} \alpha+\Delta x$ et $\bar{y}_{Z}=-Z_{0} \beta-\Delta y$.

Lorsque $\mathrm{S}$ est en $\mathrm{P}^{\prime}, \Delta x$ et $\Delta y$ s'annulent pour des valeurs de $\lambda$ respectivement voisines de 2,3 et 2,5 . Si le plan $I$ est perpendiculaire à l'axe en $P$,

$$
\Delta x \simeq 1,36 \mu \quad \text { et } \quad \Delta y \simeq 0,01 \mu
$$

avec les valeurs numériques adoptées ci-dessus. Cela correspond, dans le champ objet, à $0,08 \mu$ le long de l'axe $x$ et à une valeur infime le long de l'axe $y$.

Lorsque $S$ est en $\Omega, \Delta x$ s'annule pour $\lambda=2,3$ alors que $\Delta y$ passe par la valeur zéro pour $\lambda=3,1$. Pour $\lambda \simeq 2,5$, les valeurs de $\Delta x$ et $\Delta y$ sont voisines :

$$
|\Delta x|=2,1 R_{0} \alpha \delta=1,8 \mu
$$

et

$$
|\Delta y|=2,5 R_{0} \beta \delta=2,13 \mu \text {. }
$$

Compte tenu du grandissement, on a dans le champ objet $0,064 \mu$ en $x$ et $0,076 \mu$ en $y$. Pour $\lambda \simeq 2,80$, $|\Delta x|=4,15 R_{0} \alpha \delta \simeq 3,76 \mu$ et $|\Delta y|=0,95$ $R_{0} \beta \delta=0,81 \mu$, ce qui correspond au niveau de l'objet à $0,15 \mu$ en $x$ et $0,035 \mu$ en $y$.

Finalement, la tache entourant chaque point $E_{0}^{\prime \prime}$ du plan image a la forme d'une aigrette terminée par un arc d'ellipse. Cette aigrette est oblique lorsque $S$ est en $\boldsymbol{\Omega}$ (cas de la Fig. 13), et à peu près centrée sur l'axe $\mathrm{x}$ quand $\mathrm{S}$ est en $\mathrm{P}^{\prime}$.

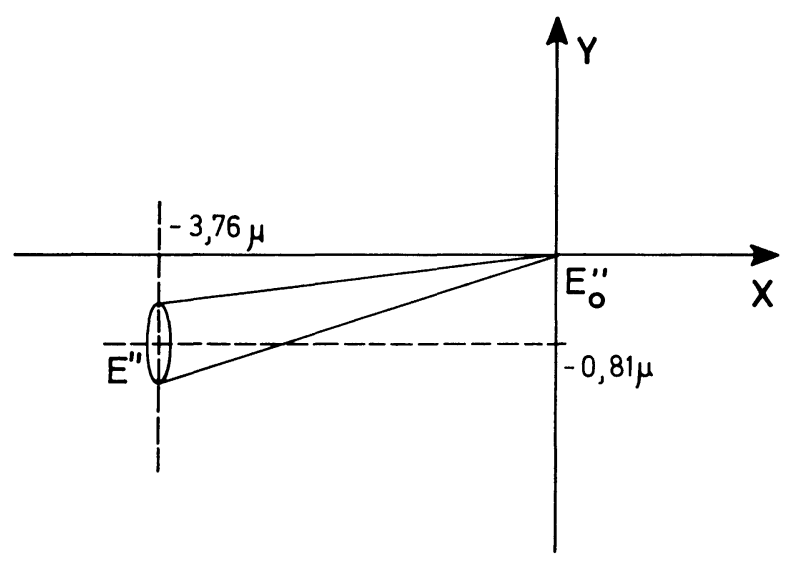

FIG. 13. - Aberrations chromatiques, $\mathrm{E}_{0}^{\prime \prime}$ point image de Gauss.

Dans l'expression de $\bar{x}_{\mathrm{Z}}$, il existe aussi un terme en $\delta^{2}$ qui provoque une légère translation parallèlement à l'axe $\mathrm{x}$ des ellipses dont l'enveloppe constitue l'aigrette, cette translation étant d'autant plus forte que la trajectoire correspond à un $\delta$ plus grand. Quelle que soit la position du miroir, les calculs sur machine montrent que le coefficient de $\delta^{2}$ relatif au champ objet reste sensiblement constant quand $\lambda$ varie entre 1,0 et 4,0.

Pour $\lambda=2,5$, l'aberration en $\delta^{2}$ est, dans le plan image de $0,47 \mu$ pour $S$ en $\Omega$ et de $0,19 \mu$ quand $S$ est en $\mathrm{P}^{\prime}$, ce qui correspond respectivement à $0,017 \mu$ et $0,011 \mu$ dans le champ objet. Lorsque $\lambda \simeq 2,8$ pour S en $\Omega$, l'aberration est de $0,39 \mu$ sur l'image, ce qui donne $0,017 \mu$ au niveau de l'objet.

Les dimensions maximum de la tache d'aberration chromatique du second ordre sur l'objet sont donc, lorsque $\mathrm{S}$ est en $\mathrm{P}^{\prime}$, avec $\lambda \simeq 2,5$ de $0,094 \mu$ en $x$ et $0,020 \mu$ en $y$, et lorsque $S$ est en $\Omega$, avec $\lambda \simeq 2,8$, de $0,16 \mu$ en $x$ et $0,051 \mu$ en $y$. On peut envisager, quand $\mathrm{S}$ 
est en $\Omega$, le cas où $\lambda \simeq 2,5$; à ce moment, les dimensions de la tache deviennent $0,083 \mu$ en $x$ et $0,091 \mu$ en $y$.

Cependant, lorsque $\mathrm{S}$ est en $\mathrm{P}^{\prime}, \bar{x}_{\mathrm{Z}}$ contient un terme du premier ordre en $\delta$, qui est prépondérant devant les aberrations du second ordre. Ce terme de dispersion est de $1,53 \mu$ dans le champ objet, ce qui est très important vis-à-vis des défauts évalués plus haut.

IV. Résultats et conclusion. - Un calcul géométrique simple et des développements limités au deuxième ordre nous ont permis de déterminer les aberrations introduites sur l'image d'un objet étendu donnée par le double prisme magnétique associé à un miroir électrostatique, pour deux valeurs particulières du rayon de ce miroir et pour une position variable de l'objet.

On aurait pu penser a priori qu'il était préférable de se placer dans le cas du stigmatisme, c'est-à-dire lorsque le sommet $\mathrm{S}$ du miroir coïncide avec le point stigmatique virtuel $\mathbf{P}^{\prime}$, mais l'étude des aberrations nous montre que, si, dans ce cas, le système est aplanétique, les défauts chromatiques interviennent d'une manière très notable. La dispersion chromatique est de $1,53 \mu$ dans le champ objet alors que la limite de résolution de la lentille à émission est de l'ordre de 1 micron; la dispersion du prisme est donc un peu supérieure au pouvoir séparateur de la lentille. On peut ainsi être amené à cette position particulière du sommet $\mathrm{S}$ au cours d'un réglage approché de l'appareil; il était donc utile de connaître la valeur des aberrations dans ce cas.

Néanmoins, comme ce calcul a été entrepris dans le but de minimiser les aberrations du système dispersif, nous avons récapitulé dans un tableau (Tableau I) les principaux termes d'aberration dans plusieurs cas : double prisme associé à un miroir convexe pour les deux positions du sommet du miroir ( $\mathrm{S}$ en $\mathrm{P}^{\prime}$ et $\mathrm{S}$ en $\Omega$ ), que nous comparons au même système associé à un miroir concave (cas de la microscopie électronique [5, 6]), ainsi qu'au cas du prisme magnétique symétrique (angles d'incidence et d'émergence de la trajectoire

TABLeAu I

(1) et (2) : : angle de $\Pi_{\mathrm{r}}^{\prime}$ et $\Pi_{a}$ nul : cas donnant le minimum d'aberrations du $2^{\mathrm{e}}$ ordre (valeurs encadrées). Dans le cas (2), la dispersion chromatique est de $1,53 \mu$; le cas (1) semble être le seul conduisant à une amélioration de la résolution.

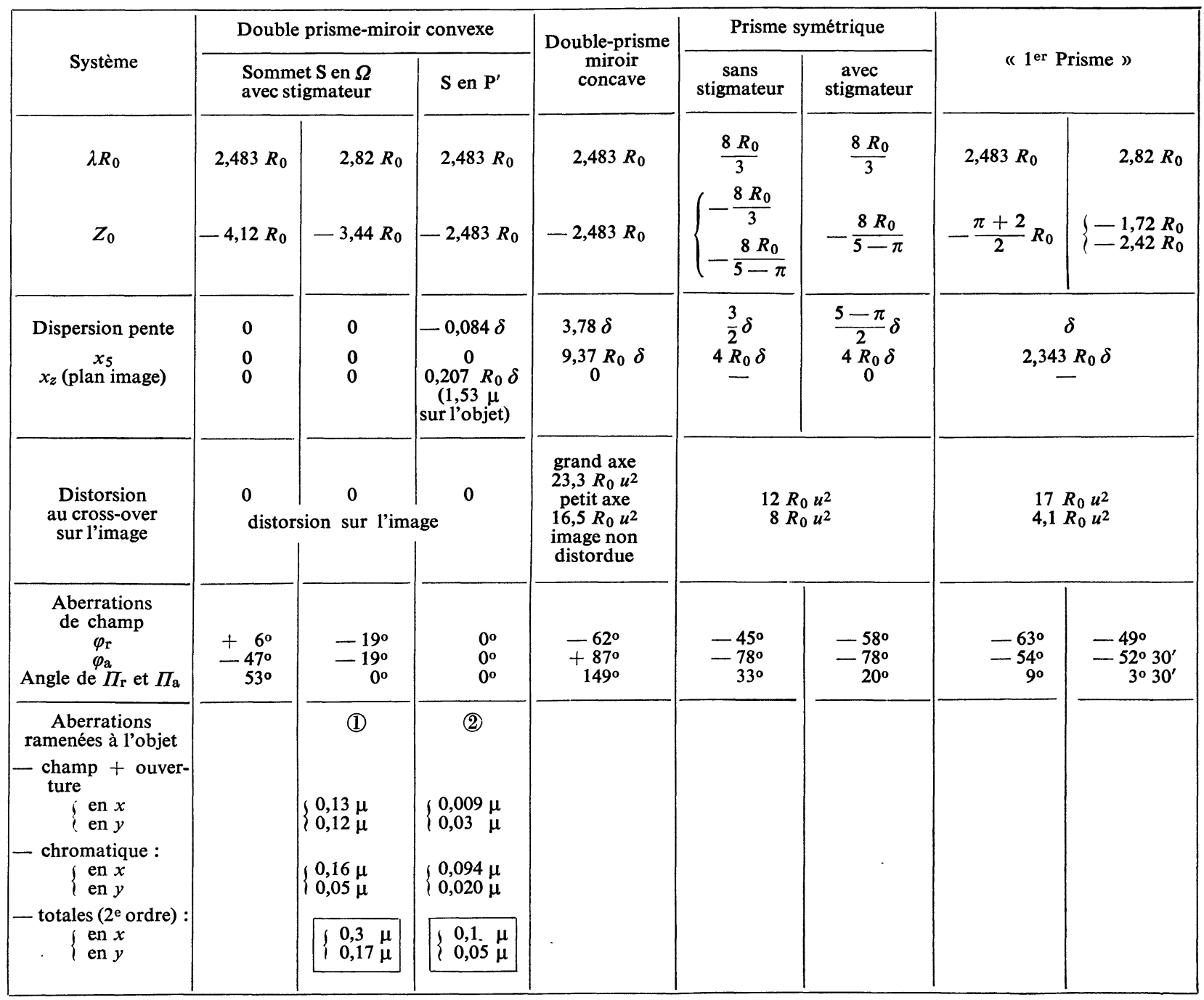


moyenne égaux) [3, 4]. Nous mentionnons aussi dans ce tableau ce qu'on obtiendrait en supposant que les ions ne subissent qu'une seule traversée du prisme (« $1^{\mathrm{er}}$ prisme » avec angle d'incidence $\varepsilon$ et émergence normale).

Nous constatons que l'astigmatisme de champ du second ordre qui se traduit par l'angle que font entre eux les plans $\Pi_{\mathrm{r}}^{\prime}$ et $\Pi_{\mathrm{a}}$, ne peut être nul que dans le cas du miroir convexe. En effet, dans le cas du miroir concave, la double traversée du prisme renforce au contraire cet astigmatisme ( $149^{\circ}$ entre les deux plans $\Pi_{\mathrm{r}}^{\prime}$ et $\Pi_{\mathrm{a}}$ au lieu de $9^{\circ}$ pour une seule traversée).

Il apparaît ainsi que la meilleure disposition possible du système serait le cas du double prisme associé à un miroir convexe centré en $\mathrm{C}^{\prime}$ et dont le sommet serait sur la focale achromatique $\Omega$. Ceci entraîne l'existence d'un astigmatisme de champ. Pour le faire disparaître, il faut donc imposer également la position de l'objet : ce dernier doit se trouver à une distance $\lambda$ du cross-over C égale à 2,82 ; il n'y a pas, alors de dispersion chromatique au premier ordre et les défauts du second ordre (chromatiques ou inclinaison de champ « résiduelle ») sont au total de l'ordre de $0,3 \mu$ sur les bords d'un champ objet de $200 \mu$ de diamètre (pour un diaphragme de contraste de $400 \mu$ et une bande d'énergie de largeur relative $\Delta V / V=1 / 400$ ).

Nous voyons ainsi que, dans ces conditions, les aberrations entachant l'image sont relativement faibles, et qu'en fait ce n'est pas le système dispersif qui limite la résolution de l'analyseur, mais la lentille à émission elle-même, dont les aberrations sont dues surtout au fait que les ions quittent la surface de l'objet avec une vitesse initiale non nulle [3], [15].

$\mathrm{Au}$ cours de ces calculs, nous avons utilisé principalement deux hypothèses simplificatrices :

- Absence de champ de fuite : l'induction magnétique est supposée uniforme à l'intérieur de l'aimant tout entier et elle s'annule brusquement à l'aplomb des faces polaires.

- Miroir exempt de toute aberration.

L'existence des champs de fuite se traduit par une modification importante de la focalisation au premier ordre, mais leur action est beaucoup moins notable sur les aberrations du second ordre dont nous pouvons penser que l'ordre de grandeur n'est que peu modifié.

Il reste donc à traiter le problème réel des trajectoires d'ions subissant l'action d'un champ de fuite, en utilisant les calculs sur machine.

D'autre part, une étude plus poussée du miroir électrostatique est nécessaire car on peut espérer une compensation de l'aberration chromatique de la lentille par celle du miroir : en effet, les particules rapides pénètrent plus profondément dans le champ du miroir et sont par conséquent soumises à son action pendant un intervalle de temps plus long que des ions plus lents. Ceci pourrait ainsi conduire à une nette amélioration de la résolution du microanalyseur ionique.

Appendice I. - ETUDE DES ABERRATIONS DU DEUXIÈME ORDRE. - Calcul géométrique des trajectoires.

Il s'agit de déterminer les trajectoires très voisines de la trajectoire principale à l'approximation du second ordre.

Nous avons effectué le calcul géométrique direct des trajectoires dans le système en divisant l'espace en cinq régions :

I. - Région sans champ magnétique avant le prisme.

II. - Couche d'entrée dans le prisme (l'induction passe de 0 à $B_{0}$ ).

III. - Région d'induction magnétique uniforme dans l'entrefer du prisme.

IV. - Couche de sortie du prisme (l'induction passe de $B_{0}$ à 0 ),

V. - Région sans champ après le prisme.

Dans les régions I et $\mathrm{V}$, les trajectoires sont rectilignes. Dans le prisme (région III), ce sont des arcs de cercle en projection sur le plan radial, des arcs d'hélice dans l'espace.

Région d'induction uniforme (région III).

Nous avons traité le calcul analytiquement dans le cas d'un prisme dont les faces font un angle quelconque avec la normale à la trajectoire (Fig. I.1) et qui fait tourner la trajectoire moyenne de $\pi / 2$ pour des particules incidentes de vitesse $v_{0}$.

Soit un ion animé de la vitesse $v$, pénétrant dans le

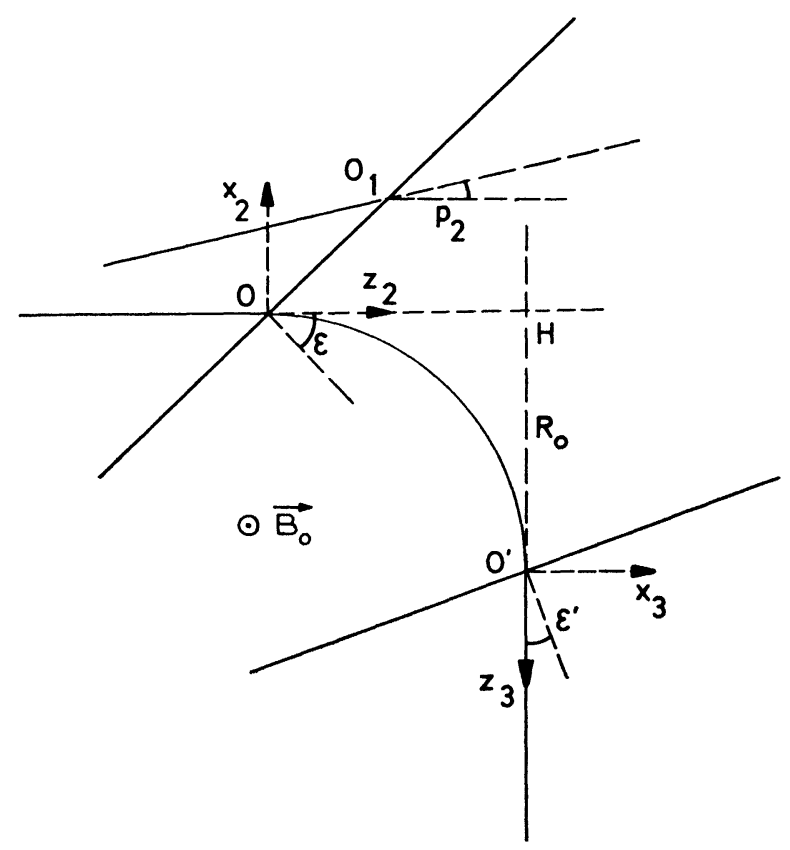

FIG. I.1. - Systèmes d'axes à l'entrée et à la sortie du prisme dans le plan radial. $\mathrm{p}_{2}$ pente de la projection sur ce plan d'une trajectoire quelconque juste après la couche d'entrée. 
prisme au point $\mathrm{J}$ de coordonnées $x_{2}, y_{2}, z_{2}$ $\left(z_{2}=x_{2} \operatorname{tg} \varepsilon\right)$ dans le système d'axes $O x_{2} y_{2} z_{2}$ lié à la face d'entrée (l'axe $z$ est orienté dans le sens du mouvement et le point $\mathbf{J}$ se projette en $\mathrm{O}_{1}$ sur le plan radial : Fig. I.1).

Sa trajectoire, immédiatement après la couche d'entrée, fait, en projection sur les sections principales, les angles $p_{2}$ et $q_{2}$ avec la trajectoire principale; elle s'incurve dans le prisme en un arc de cercle en projection sur le plan radial, de rayon $R^{\prime}=\mathrm{R} \cos q_{2}$ où $R=\mathrm{m} v / q B_{0}$.

Si on considère que la vitesse $v$ d'un ion peut différer de la vitesse $v_{0}$ d'un infiniment petit du premier ordre en $\delta$, c'est-à-dire que $v=v_{0}(1+\delta)$ où $\delta=\Delta v / v_{0}$, le rayon de la trajectoire correspondante du plan radial sera $R=R_{0}(1+\delta)$ avec $R_{0}=m v_{0} / q B_{0}$, rayon de la trajectoire principale. Nous pourrons ainsi obtenir, dans le plan de la face de sortie, les variations des coordonnées d'intersection d'une trajectoire quelconque, introduites par le terme $\delta$.

L'intersection du cercle tangent en $\mathrm{O}_{1}$ à la droite de pente $p_{2}$, de rayon $\mathrm{R}^{\prime}$, avec la face de sortie du prisme de pente $\operatorname{tg} \varepsilon^{\prime}=t^{\prime}$, donnera les coordonnées $x_{3}$, $z_{3}$, du point de sortie en fonction des coordonnées $x_{2}$, $z_{2}$, du point d'entrée. La pente $\mathrm{p}_{3}$ de la trajectoire à la sortie du prisme, immédiatement avant la couche de passage, est donnée par la position de la tangente au cercle. Comme la trajectoire est un arc d'hélice, la «pente axiale » reste invariante et $q_{3}=q_{2}$. La cote du point de sortie est $y_{3}=y_{2}+R^{\prime} \omega q_{2}$ si $\omega$ est l'angle dont a tourné la projection de la trajectoire sur le plan radial : $\omega=\pi / 2+p_{2}-p_{3}$. Compte tenu de l'équation de la face d'entrée $z_{2}=x_{2} t$, on trouve :

- pour la première section principale

$$
\begin{aligned}
x_{3}= & x_{2} t+R_{0} p_{2}+R_{0} \delta-\frac{x_{2}^{2}}{2 R_{0}}\left(1-t t^{\prime}\right)^{2}+ \\
& +t^{\prime} x_{2} p_{2}\left(1-t t^{\prime}\right)+x_{2} \delta\left(1+t^{\prime}\right)\left(1-t t^{\prime}\right) \\
& -R_{0} \frac{t^{\prime 2}}{2} p_{2}^{2}+R_{0} p_{2} \delta\left(1-t^{\prime}-t^{\prime 2}\right) \\
& -R_{0} \frac{q_{2}^{2}}{2}-R_{0} \frac{\delta^{2}}{2}\left(1+t^{\prime}\right)^{2}
\end{aligned}
$$$$
p_{3}=-\frac{x_{2}}{R_{0}}\left(1-t t^{\prime}\right)+t^{\prime} p_{2}+\delta\left(1+t^{\prime}\right)
$$$$
-\frac{x_{2}^{2}}{2 R_{0}^{2}} t^{\prime}\left(1-t t^{\prime}\right)^{2}+t^{\prime 2} \frac{x_{2} p_{2}}{R_{0}}\left(1-t t^{\prime}\right)
$$$$
+\frac{x_{2} \delta}{R_{0}} t^{\prime}\left(1+t^{\prime}\right)\left(1-t t^{\prime}\right)
$$$$
-\frac{p_{2}^{2}}{2}\left(1+t^{\prime 3}\right)+p_{2} \delta t^{\prime}\left(1-t^{\prime}-t^{\prime 2}\right)
$$$$
-\frac{\delta^{2}}{2} t^{\prime}\left(1+t^{\prime}\right)-\frac{q_{2}^{2}}{2}\left(1+t^{\prime}\right)
$$

- pour la deuxième section principale

$$
\begin{aligned}
y_{3}=y_{2} & +R_{0} \frac{\pi}{2} q_{2}+x_{2} q_{2}\left(1-t t^{\prime}\right)+ \\
& +R_{0}\left(1-t^{\prime}\right) p_{2} q_{2}+R_{0} q_{2} \delta\left(\frac{\pi}{2}-1-t^{\prime}\right) \\
q_{3}=q_{2} . &
\end{aligned}
$$

\section{Régions II et IV.}

Le problème est maintenant de déterminer l'action des couches de passage sur des trajectoires situées hors $\mathrm{du}$ plan de symétrie dans le cas où $\varepsilon$ n'est pas nul. Ces régions sont des zones de discontinuité pour l'induction. Nous avons supposé, pour faire le calcul, que ces régions de transition étaient infiniment minces, et que l'induction magnétique passait brutalement de la valeur zéro à une valeur finie $B_{0}$ (ou vice versa).

Une telle couche de passage se manifeste par l'existence d'une induction magnétique transversale $\mathbf{B}_{t}$, perpendiculaire à la face du prisme et parallèle au plan de symétrie (Fig. 6). L'induction $B_{\mathrm{t}}$ est proportionnelle à la cote $y$ du point d'entrée $J$ de la trajectoire dans le prisme, et à la dérivée normale de la composante suivant $y, B_{y}$ de l'induction. $\mathbf{B}_{\mathbf{t}}$ est dirigée vers l'intérieur de l'aimant pour des points de cote y positive, en sens inverse pour des points de cote $y$ négative (l'axe $y$ étant de même sens que $\mathbf{B}_{0}$ ) :

$$
B_{\mathrm{t}}=y \frac{\mathrm{d} B}{\mathrm{~d} n} .
$$

L'induction $\mathbf{B}_{\mathbf{t}}$ qui existe exclusivement dans la zone de passage, $\mathbf{y}$ donne naissance à une force $\mathbf{F}_{\boldsymbol{t}}$ contenue dans le plan de la région de discontinuité, c'est-à-dire dans la face du prisme. Une particule arrivant à la vitesse $\mathbf{v}$ dans la zone de passage, est soumise à la force $\mathbf{F}_{\mathbf{t}}=q \mathbf{v}_{\wedge} \mathbf{B}_{\mathrm{t}}$. Soit $V$ la projection de la vitesse $v$ sur la face du prisme : $F_{t}=q V B_{t} . F_{t}$ est orthogonale au vecteur $\mathbf{V}$ qui conserve un module constant, comme le montre d'ailleurs l'intégration des équations du mouvement en projection sur des axes contenus dans la face d'entrée (ou de sortie) du prisme.

$$
F_{\mathrm{t}}=q V B_{\mathrm{t}}=m \frac{V^{2}}{\rho} \quad \text { avec } \quad \rho=\frac{\mathrm{d} s}{\mathrm{~d} \theta}
$$

rayon de courbure de la trajectoire en projection sur la face du prisme.

$$
\mathrm{d} \theta=\frac{q y}{m V} \mathrm{~d} B \frac{\mathrm{d} s}{\mathrm{~d} n} .
$$

Or

$$
\frac{\mathrm{d} s}{\mathrm{~d} n}=\frac{\mathrm{d} s}{\mathrm{~d} t} \cdot \frac{\mathrm{d} t}{\mathrm{~d} n}=\frac{V}{V_{\mathrm{n}}}
$$

( $V_{\mathrm{n}}$ composante de la vitesse sur la normale à la face d'entrée XY du prisme).

D'où

$$
\mathrm{d} \theta=\frac{q y}{m V_{\mathrm{n}}} \mathrm{d} B .
$$

La trajectoire, donc le vecteur $\mathbf{V}$, tourne d'un angle 
$\mathrm{d} \theta$ quand l'induction varie de $\mathrm{d} B$, mais le rayon de courbure $\rho$ étant infiniment petit à la limite (couche de passage infiniment mince), tout se passe comme si $\mathbf{V}$ recevait une impulsion et tournait d'un angle fini

$$
\Delta \theta=\int_{0}^{B_{0}} \frac{q y}{m V_{\mathrm{n}}} \mathrm{d} B
$$

passant de la position $\mathbf{V}_{1}$ à l'entrée de la couche à la position $\mathbf{V}_{2}$ à la sortie (Fig. I.2). Etant donné l'hypo-

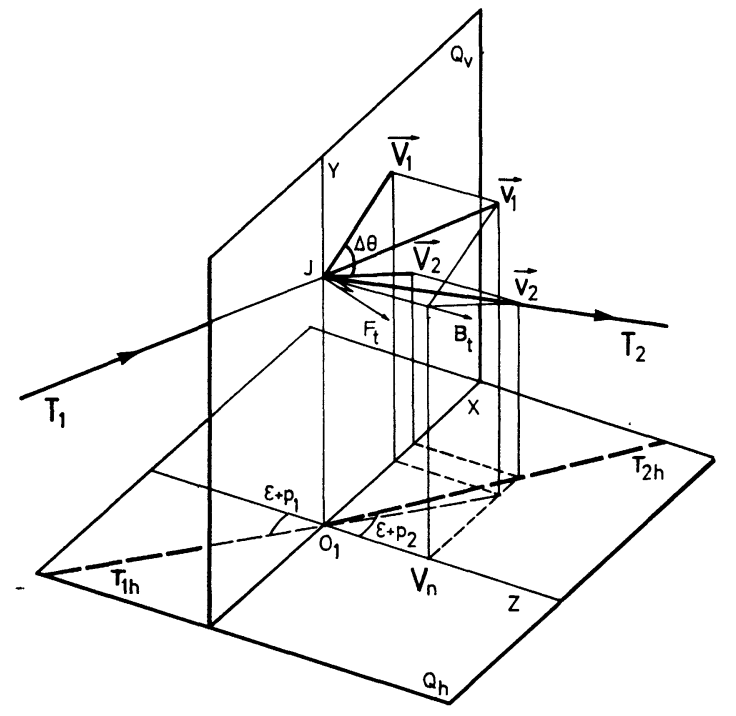

FIG. I.2. - Action d'une couche de passage infiniment mince schématisée par le plan $Q_{v}$ perpendiculaire au plan radial $Q_{h}$. $\mathrm{T}_{1} \mathrm{~J}$ trajectoire incidente de projection $T_{1 h} \mathrm{O}_{1}$ sur $Q_{h}$, vitesse $\mathrm{v}_{1}$ de projection $V_{1}$ sur $Q_{h}$.

$\mathrm{JT}_{2}$ trajectoire immédiatement après la couche de passage ; $\mathrm{O}_{1} \mathrm{~T}_{2 \mathrm{~h}}$ projection de $\mathrm{JT}_{2}$ sur $Q_{h}$; vitesse $\mathrm{v}_{2}$ de projection $V_{2}$ sur la face d'entrée $Q_{v}$.

$\mathbf{B}_{t}$ induction transversale normale à $Q_{v} ; \mathbf{F}_{\mathrm{t}}$ (contenue dans $Q_{v}$ ) : force due à $B_{t} . V_{n}$ composante de $v_{1}$ et $v_{2}$ sur la normale à $Q v$. $\Delta \theta$ angle de $v_{1}$ et $v_{2}$.

thèse de la couche sans épaisseur, les coordonnées de l'intersection de la trajectoire avec la face du prisme $\mathrm{XY}$, restent invariantes, donc y est constant et

$$
\Delta \theta=\frac{q y}{m V_{\mathrm{n}}} \int_{0}^{B_{0}} \mathrm{~d} B=\frac{q y}{m V_{\mathrm{n}}} B_{0} .
$$

Le rayon de courbure dans l'aimant est $R=m v / q B_{0}$, d'où :

$$
\Delta \theta=\frac{y v}{R V_{\mathrm{n}}} .
$$

L'axe du système correspond à des ions de vitesse $v_{0}$ décrivant un arc de cercle de rayon $R_{0}=m v_{0} / q B_{0}$. Si $v=v_{0}(1+\delta)$, la déviation subie par la trajectoire dépend de $\delta$ :

$$
\Delta \theta=\frac{y v}{R_{0}(1+\delta) V_{\mathrm{n}}}=\frac{y}{R_{0}(1+\delta)} \frac{\mathrm{v}_{0}}{V_{0 \mathrm{n}}}=\frac{\Delta \theta_{0}}{1+\delta} .
$$

Action de la couche de passage sur les pentes d'une trajectoire. - Soient $p_{1}$ et $q_{1}$ les pentes, à l'entrée de la couche, d'une trajectoire qui rencontre la face d'entrée en un point de coordonnées $x_{1}, y_{1}$ et soient $p_{2}$ et $q_{2}$ les pentes à la sortie. La rotation de $\Delta \theta$ du vecteur $\mathbf{V}$ se traduit, dans le cas de l'entrée dans le prisme lors de la première traversée, par la relation, pour la «pente axiale» :

$$
q_{2}=q_{1}-\frac{y_{1}}{R} \operatorname{tg}\left(p_{1}+\varepsilon\right) .
$$

Avec tg $\varepsilon=t$ et $R=R_{0}(1+\delta)$, nous obtenons

ou

$$
q_{2}=q_{1}-\frac{y_{1} t}{R}-\frac{y_{1} p_{1}}{R}\left(1+t^{2}\right)
$$

$$
q_{2}=q_{1}-\frac{y_{1} t}{R_{0}}-\frac{y_{1} p_{1}}{R_{0}}\left(1+t^{2}\right)+y_{1} \delta \frac{t}{R_{0}} .
$$

Il s'introduit un terme du premier ordre, fonction de la cote $y_{1}$ du point d'entrée, qui traduit le phénomène de focalisation axiale par la face d'entrée du prisme, inclinée de $(\pi / 2-\varepsilon)$ sur l'axe principal (Fig. I.2). La face d'entrée joue ainsi, dans la seconde section secondaire correspondant à $p_{1}$, le rôle d'une lentille convergente dont la convergence varie avec la vitesse des particules (aberration chromatique des lentilles). Si $\varepsilon$ est nul (face perpendiculaire à l'axe), il n'y a plus de focalisation axiale.

Pour la «pente radiale», l'action de la couche de passage se traduit par:

$p_{2}=p_{1}+\frac{y_{1} q_{1}}{R}-\frac{y_{1}^{2} t}{2 R^{2}}=p_{1}+\frac{y_{1} q_{1}}{R_{0}}-\frac{y_{1}^{2} t}{2 R_{0}^{2}}$

au troisième ordre près. Comme nous nous bornons aux termes du second ordre, la variation de la vitesse des particules n'a pas d'influence sur $p_{2}$. Il apparaît dans l'expression de $p_{2}$ un terme du second ordre fonction de la "pente axiale " $q_{1}$ et de la cote $y_{1}$. En plus de l'effet de focalisation, une trajectoire située en dehors du plan de symétrie reçoit donc une impulsion qui a pour effet de la faire sortir de la seconde section secondaire correspondant à la pente $p_{1}$ (Fig. I.3). Ceci a déjà été souligné par Enge [14].

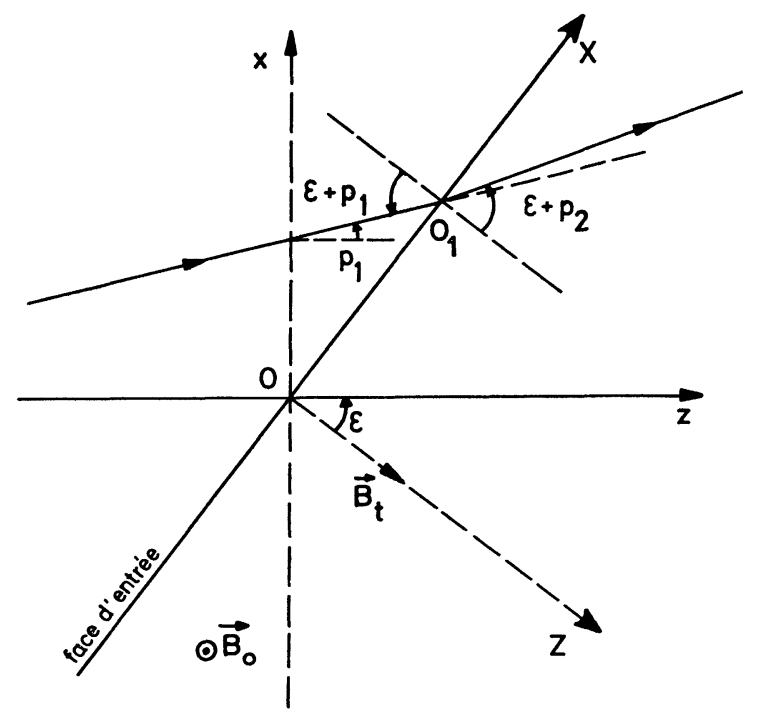

Fig. I.3. - Action de la couche de passage : projection sur le plan radial. 
Le calcul serait analogue pour la face de sortie du prisme. Nous pouvons alors récapituler les diverses formules de transformation traduisant l'action de chacune des régions :
Première traversée : $t, t^{\prime}=0$ (sortie perpendiculaire pour la trajectoire moyenne); distance $\mathrm{CO}=R_{0} l$.
Deuxième traversée : $t=0, t^{\prime}$ (entrée normale pour la trajectoire moyenne); distance $\mathrm{C}^{\prime} \mathrm{O}^{\prime}=R_{0} \bar{l}$.

Région I (sans champ magnétique)

$$
\begin{aligned}
& x_{1}=x_{0}+R_{0} l p_{0}+x_{0} p_{0} t+R_{0} l t p_{0}^{2} \\
& y_{1}=y_{0}+R_{0} l q_{0}+x_{0} q_{0} t+R_{0} l t p_{0} q_{0} \\
& z_{1}=x_{1} t \\
& p_{1}=p_{0}, q_{1}=q_{0}
\end{aligned}
$$

$$
\begin{aligned}
& \bar{x}_{1}=\bar{x}_{0}+R_{0} \bar{l} \bar{p}_{0} \\
& \bar{y}_{1}=\bar{y}_{0}+R_{0} \bar{l} \bar{q}_{0} \\
& \bar{z}_{1}=0 \\
& \bar{p}_{1}=\bar{p}_{0}, \bar{q}_{1}=\bar{q}_{0} .
\end{aligned}
$$

Région II : couche d'entrée dans le prisme

$$
\begin{array}{l|l}
x_{2}=x_{1}, y_{2}=y_{1}, z_{2}=z_{1} & \bar{x}_{2}=\bar{x}_{1}, \bar{y}_{2}=\bar{y}_{1}, \bar{z}_{2}=\bar{z}_{1}=0 \\
p_{2}=p_{1}+\frac{y_{1} q_{1}}{R_{0}}-\frac{y_{1}^{2} t}{2 R_{0}^{2}} & \bar{p}_{2}=\bar{p}_{1}+\frac{\bar{y}_{1} \bar{q}_{1}}{R_{0}} \\
q_{2}=q_{1}-\frac{y_{1} t}{R_{0}}-\frac{y_{1} p_{1}}{R_{0}}\left(1+t^{2}\right)+\frac{y_{1} t \delta}{R_{0}} & \bar{q}_{2}=\bar{q}_{1}-\frac{\bar{y}_{1} \bar{p}_{1}}{R_{0}} .
\end{array}
$$

Région III : champ uniforme

$$
\begin{gathered}
x_{3}=x_{2} t+R_{0} p_{2}+R_{0} \delta-x_{2}^{2} \frac{1}{2 R_{0}}+x_{2} \delta \\
+R_{0} p_{2} \delta-R_{0} \frac{q_{2}^{2}}{2}-R_{0} \frac{\delta^{2}}{2} \\
p_{3}=-\frac{x_{2}}{R_{0}}+\delta-\frac{p_{2}^{2}}{2}-\frac{q_{2}^{2}}{2} \\
y_{3}=y_{2}+R_{0} \frac{\pi}{2} q_{2}+x_{2} q_{2}+R_{0} p_{2} q_{2} \\
+R_{0} q_{2} \delta\left(\frac{\pi}{2}-1\right)
\end{gathered}
$$

$q_{3}=q_{2}$

$$
\begin{aligned}
\bar{x}_{3}= & R_{0} \bar{p}_{2}+R_{0} \delta-\frac{\bar{x}_{2}^{2}}{2 R_{0}}+t^{\prime} \bar{x}_{2} \bar{p}_{2}+ \\
& +\bar{x}_{2} \delta\left(1+t^{\prime}\right)-R_{0} \frac{t^{\prime 2}}{2} \bar{p}_{2} \\
& +R_{0} \bar{p}_{2} \delta\left(1-t^{\prime}-t^{\prime 2}\right)-R_{0} \frac{\bar{q}_{2}^{2}}{2} \\
& -R_{0} \frac{\delta^{2}}{2}\left(1+t^{\prime}\right)^{2} \\
\bar{p}_{3}= & -\frac{\bar{x}_{2}}{R_{0}}+t^{\prime} \bar{p}_{2}+\delta\left(1+t^{\prime}\right)-\frac{\bar{x}_{2}^{2} t^{\prime}}{2 R_{0}^{2}} \\
& +\frac{\bar{x}_{2} \bar{p}_{2}}{R_{0}} t^{\prime 2}+\frac{\bar{x}_{2} \delta}{R_{0}} t^{\prime}\left(1+t^{\prime}\right) \\
& -\frac{\bar{p}_{2}^{2}}{2}\left(1+t^{\prime 3}\right)-\frac{\bar{q}_{2}^{2}}{2}\left(1+t^{\prime}\right) \\
& +\bar{p}_{2} \delta t^{\prime}\left(1-t^{\prime}-t^{\prime 2}\right)-\frac{\delta^{2}}{2} t^{\prime}\left(1+t^{\prime}\right)^{2} \\
\bar{y}_{3}= & \bar{y}_{2}+R_{0} \frac{\pi}{2} \bar{q}_{2}+\bar{x}_{2} \bar{q}_{2}+R_{0}\left(1-t^{\prime}\right) \bar{p}_{2} \bar{q}_{2} \\
& +R_{0} \bar{q}_{2} \delta\left(\frac{\pi}{2}-1-t^{\prime}\right) \\
\bar{q}_{3}= & \bar{q}_{2} \cdot
\end{aligned}
$$


Région IV : couche de sortie

$$
\begin{aligned}
& x_{4}=x_{3}, y_{4}=y_{3}, z_{4}=z_{3}=0 \\
& p_{4}=p_{3}-\frac{y_{3} q_{3}}{R_{0}} \\
& q_{4}=q_{3}+\frac{y_{3} p_{3}}{R_{0}}
\end{aligned}
$$

$$
\begin{aligned}
& \bar{x}_{4}=\bar{x}_{3}, \bar{y}_{4}=\bar{y}_{3}, \bar{z}_{4}=\bar{z}_{3} \\
& \bar{p}_{4}=\bar{p}_{3}+\bar{y}_{3}^{2} \frac{t^{\prime}}{2 R_{0}^{2}}-\frac{\bar{y}_{3} \bar{q}_{3}}{R_{0}} \\
& \bar{q}_{4}=\bar{q}_{3}-\bar{y}_{3} \frac{t^{\prime}}{R_{0}}+\frac{\bar{y}_{3} \bar{p}_{3}}{R_{0}}\left(1+t^{\prime 2}\right)+\frac{\bar{y}_{3} \delta t^{\prime}}{R_{0}}
\end{aligned}
$$

Région V (sans champ magnétique)

Cross-over à la distance $R_{0} l^{\prime}$ de $\mathrm{O}^{\prime}$

$$
\begin{aligned}
& x_{5}=x_{4}+R_{0} l^{\prime} p_{4} \\
& y_{5}=y_{4}+R_{0} l^{\prime} q_{4} \\
& p_{5}=p_{4}, q_{5}=q_{4}
\end{aligned}
$$

On pourrait d'ailleurs mettre toutes ces expressions sous forme de matrices de passage comportant les termes du second ordre responsables des aberrations. La combinaison de ces relations (ou le produit des matrices successives) permet d'exprimer les coordon-
Cross-over à la distance $R_{0} \bar{l}^{\prime}$ de $\mathrm{O}^{\prime \prime}$

$$
\begin{aligned}
& \bar{x}_{5}=\bar{x}_{4}+R_{0} \bar{l}^{\prime} \bar{p}_{4}+\bar{x}_{4} \bar{p}_{4} t^{\prime} \\
& \bar{y}_{5}=\bar{y}_{4}+R_{0} \bar{l}^{\prime} \bar{q}_{4}+\bar{x}_{4} \bar{q}_{4} t^{\prime} \\
& \bar{p}_{5}=\bar{p}_{4}, \bar{q}_{5}=\bar{q}_{4} .
\end{aligned}
$$

nées de l'intersection d'une trajectoire avec le plan de la pupille de sortie du prisme en fonction des coordonnées $x_{0}, y_{0}$, de l'intersection de cette trajectoire avec le plan de la pupille d'entrée et en fonction des pentes initiales $p_{0}$ et $q_{0}$.

Première traversée :

$$
\begin{aligned}
& \mathrm{CO}=l R_{0}=2 R_{0} ; \quad \mathrm{O}^{\prime} \mathrm{C}^{\prime}=R_{0}\left(t+\frac{1}{2}\right) ; t^{\prime}=0 \\
& x_{5}=-\frac{x_{0}}{2}+0 R_{0} p_{0}+R_{0} \delta\left(t+\frac{3}{2}\right)+x_{0} \delta+3 R_{0} p_{0} \delta-R_{0} \frac{\delta^{2}}{2}-\frac{x_{0}^{2}}{2 R_{0}}-x_{0} p_{0}\left(2+\frac{t}{2}\right) \\
& -R_{0} p_{0}^{2}\left(\frac{9}{4}+\frac{3 t}{2}\right)+\frac{y_{0}^{2}}{R_{0}}\left[\frac{t^{2}}{4}(1-\pi)-\frac{t^{3}}{2}(\pi+1)\right]+y_{0} q_{0}\left[\frac{1+t+\pi t}{2}+2 t^{2}-2 t^{3}-2 \pi t^{3}\right] \\
& +R_{0} q_{0}^{2}\left[\frac{1-\pi}{4}+\frac{t}{2}(1+\pi)+t^{2}(3+\pi)-2 t^{3}(1+\pi)\right] \\
& p_{5}=-\frac{x_{0}}{R_{0}}-2 p_{0}+\delta-x_{0} p_{0} \frac{t}{R_{0}}-p_{0}^{2}\left[\frac{1+4 t}{2}\right]+\frac{y_{0}^{2}}{R_{0}^{2}}\left[t\left(1-\frac{\pi t}{2}\right)-\frac{t^{2}}{2}\right] \\
& +\frac{y_{0} q_{0}}{R_{0}}\left(-1+5 t+\pi t-2 t^{2}-2 \pi t^{2}\right)+q_{0}^{2} t(2 t-1)^{2} \\
& y_{5}=-\frac{y_{0}}{2}\left(t+\frac{\pi+1}{2}\right)+0 R_{0} q_{0}+y_{0} \delta\left[\frac{1}{2}+t\left(\frac{5}{2}-\frac{\pi}{4}\right)+t^{2}\left(1-\frac{\pi}{2}\right)\right] \\
& +R_{0} q_{0} \delta\left[\frac{3 \pi}{4}+5 t+2 t^{2}-\pi t^{2}\right]-\frac{x_{0} y_{0}}{R_{0}}\left(\frac{1}{2}+2 t-\frac{\pi t}{4}-\frac{\pi t^{2}}{2}\right)-x_{0} q_{0}\left(\frac{\pi}{4}+3 t+\frac{t^{2}}{2}-\frac{\pi t^{2}}{2}+t^{3}\right) \\
& -\frac{y_{0} p_{0}}{2}\left(3+\pi+12 t-\pi t+t^{2}-\pi t^{2}+2 t^{3}\right)-R_{0} p_{0} q_{0}\left(\frac{3 \pi}{2}+10 t+2 t^{2}+4 t^{3}\right) \\
& q_{5}=-\frac{y_{0} t}{R_{0}}+q_{0}(1-2 t)+\frac{y_{0} \delta}{R_{0}}\left(1+t-\frac{\pi t}{2}\right)+q_{0} \delta\left[2+2 t+\frac{\pi}{2}(1-2 t)\right]-\frac{x_{0} y_{0}}{R_{0}^{2}}\left(1-\frac{\pi t}{2}\right) \\
& -\frac{x_{0} q_{0}}{R_{0}}\left(2+\frac{\pi}{2}-\pi t+t^{2}\right)-\frac{y_{0} p_{0}}{R_{0}}\left(3-\pi t+t^{2}\right)-2 p_{0} q_{0}\left(3+\frac{\pi}{2}-\pi t+2 t^{2}\right) \text {. }
\end{aligned}
$$

Avec $t=0,843$.

$$
\begin{aligned}
x_{5}= & -0,5 x_{0}+2,343 R_{0} \delta+x_{0} \delta+3 R_{0} p_{0} \delta-0,5 R_{0} \delta^{2}-0,5 \frac{x_{0}^{2}}{R_{0}}-2,42 x_{0} p_{0} \\
& -3,52 R_{0} p_{0}^{2}-1,62 \frac{y_{0}^{2}}{R_{0}}-1,30 y_{0} q_{0}+0,610 R_{0} q_{0}^{2}
\end{aligned}
$$




$$
\begin{aligned}
p_{5}= & -\frac{x_{0}}{R_{0}}-2 p_{0}+\delta-0,843 \frac{x_{0} p_{0}}{R_{0}}-2,19 p_{0}^{2}-0,629 \frac{y_{0}^{2}}{R_{0}^{2}}-0,022 \frac{y_{0} q_{0}^{2}}{R_{0}}+0,397 q_{0}^{2} \\
y_{5}= & -1,457 y_{0}+1,54 y_{0} \delta+5,76 R_{0} q_{0} \delta-0,408 \frac{x_{0} y_{0}}{R_{0}}-3,15 x_{0} q_{0}-6,64 y_{0} p_{0}-17,0 R_{0} p_{0} q_{0} \\
q_{5}= & -0,843 \frac{y_{0}}{R_{0}}-0,686 q_{0}+0,519 \frac{y_{0} \delta}{R_{0}}+2,61 q_{0} \delta+0,325 \frac{x_{0} y_{0}}{R_{0}^{2}}-1,63 \frac{x_{0} q_{0}}{R_{0}} \\
& -1,06 \frac{y_{0} p_{0}}{R_{0}}-6,69 p_{0} q_{0} .
\end{aligned}
$$

Deuxième traversée :

$$
\mathrm{C}^{\prime} \mathrm{O}^{\prime}=\bar{l} R_{0}=R_{0}\left(t+\frac{1}{2}\right) ; \quad \mathrm{O}^{\prime \prime} \mathrm{C}^{\prime \prime}=\bar{l}^{\prime} R_{0}=2 R_{0} .
$$

Dans le plan de la pupille de sortie :

$$
\begin{aligned}
& \bar{x}_{5}=-2 \bar{x}_{0}+R_{0} \delta(3+2 t)+\bar{x}_{0} \delta\left(1+2 t+2 t^{2}\right)+R_{0} \bar{p}_{0} \delta\left(\frac{3}{2}+4 t+2 t^{2}\right)-R_{0} \frac{\delta^{2}}{2}\left(1+t+t^{2}+t^{3}\right) \\
&-\frac{\bar{x}_{0}^{2}}{R_{0}}\left(t+\frac{1}{2}\right)-\bar{x}_{0} \bar{p}_{0}\left(\frac{1}{2}+2 t\right)-\frac{R_{0} \bar{p}_{0}^{2}}{2}\left(\frac{9}{4}+\frac{3 t}{2}\right)+\frac{\bar{y}_{0}^{2} t}{R_{0}}+\bar{y}_{0} \bar{q}_{0}\left(-1+3 t+\pi t+2 t^{2}\right) \\
&+R_{0} \bar{q}_{0}^{2}\left[-2-\pi-t+2 t^{2}+t\left(t+\frac{\pi+1}{2}\right)^{2}\right] \\
& \bar{p}_{5}=-\frac{\bar{x}_{0}}{R_{0}}-\frac{\bar{p}_{0}}{2}+\delta(1+t)+\frac{\bar{x}_{0} \delta}{R_{0}} t(1+t)+\bar{p}_{0} \delta t\left(\frac{3+t}{2}\right)-\delta^{2} \frac{t(1+t)^{2}}{2}-\frac{t \bar{x}_{0}^{2}}{2 R_{0}^{2}} \\
&-\bar{x}_{0} \bar{p}_{0} \frac{t}{2 R_{0}}-\frac{\bar{p}_{0}^{2}}{2}\left(1+\frac{t}{4}\right)+\frac{t \bar{y}_{0}^{2}}{2 R_{0}^{2}}+\bar{y}_{0} \bar{q}_{0}\left(-1+\frac{3 t}{2}+\frac{\pi t}{2}+t^{2}\right) \\
&+\frac{\bar{q}_{0}^{2}}{2}\left[-2-\pi-2 t+2 t^{2}+t\left(t+\frac{\pi+1}{2}\right)^{2}\right] \\
& \bar{y}_{5}= \bar{y}_{0}(1-2 t)+\bar{y}_{0} \delta\left(2+4 t+t^{2}+2 t^{3}\right)+R_{0} \bar{q}_{0} \delta\left(\frac{5}{2}+2 \pi+\frac{17 t}{2}+\frac{\pi t}{2}+7 t^{2}\right) \\
&-2 \frac{\bar{x}_{0} \bar{y}_{0}}{R_{0}}\left(1+t^{2}\right)-\bar{x}_{0} \bar{q}_{0}\left[\pi+4 t+2 t^{2}\left(t+\frac{\pi+1}{2}\right)\right]-\bar{y}_{0} \bar{p}_{0}\left(3+\frac{\pi}{2}-\pi t+2 t^{2}\right)- \\
&+R_{0} \bar{p}_{0} \bar{q}_{0}\left[\frac{1}{2}-\frac{\pi}{4}-4 t+\pi t^{2}-\left(1+2 t^{2}\right)\left(t+\frac{\pi+1}{2}\right)\right] \\
& \bar{q}_{5}=-\bar{y}_{0} \frac{t}{R_{0}}+\bar{q}_{0}\left[1-t\left(t+\frac{\pi+1}{2}\right)\right]+\frac{\bar{y}_{0} \delta}{R_{0}}\left(1+2 t+t^{2}+t^{3}\right)+\bar{q}_{0} \delta\left[\frac{\pi+1}{2}+3 t+\frac{\pi t}{2}+\frac{7 t^{2}}{2}\right. \\
&\left.+\frac{\pi}{2} t^{2}+\frac{3}{2} t^{3}+\frac{\pi}{2} t^{3}+t^{4}\right]-\frac{\bar{x}_{0} \bar{y}_{0}}{R_{0}^{2}}\left(1+t^{2}\right)-\frac{\bar{x}_{0} \bar{q}_{0}}{R_{0}}\left[t+\left(1+t^{2}\right)\left(t+\frac{\pi+1}{2}\right)\right] \\
&-\frac{\bar{y}_{0} \bar{p}_{0}}{R_{0}}\left(\frac{3}{2}-\frac{\pi t}{2}+\frac{t^{2}}{2}\right)-\frac{\bar{p}_{0} \bar{q}_{0}}{2}\left[1+5 t-\frac{\pi t}{2}-\pi t^{2}+\left(1+t^{2}\right)\left(t+\frac{\pi+1}{2}\right)\right] . \\
&(\mathrm{I}
\end{aligned}
$$

Numériquement :

$$
\begin{aligned}
\bar{x}_{5}= & -2 \bar{x}_{0}+4,686 R_{0} \delta+4,11 \bar{x}_{0} \delta+6,29 R_{0} \bar{p}_{0} \delta-1,58 R_{0} \delta^{2}-1,34 \frac{\bar{x}_{0}^{2}}{R_{0}} \\
& -2,19 \bar{x}_{0} \bar{p}_{0}-1,76 R_{0} \bar{p}_{0}^{2}+0,843 \frac{\bar{y}_{0}^{2}}{R_{0}}+5,60 \bar{y}_{0} \bar{q}_{0}+2,60 R_{0} \bar{q}_{0}^{2} \\
\bar{p}_{5}= & -\frac{\bar{x}_{0}}{R_{0}}-0,5 \bar{p}_{0}+1,843 \delta+1,55 \frac{\bar{x}_{0} \delta}{R_{0}}+1,62 \bar{p}_{0} \delta-1,43 \delta^{2}-0,422 \frac{\bar{x}_{0}^{2}}{R_{0}^{2}} \\
& -0,422 \frac{\bar{x}_{0} \bar{p}_{0}}{R_{0}}-0,605 \bar{p}_{0}^{2}+0,422 \frac{\bar{y}_{0}^{2}}{R_{0}^{2}}+2,30 \frac{\bar{y}_{0} \bar{q}_{0}}{R_{0}}+0,877 \bar{q}_{0}^{2}
\end{aligned}
$$




$$
\begin{aligned}
\bar{y}_{5}= & -0,686 \bar{y}_{0}+7,28 \bar{y}_{0} \delta+22,2 R_{0} \bar{q}_{0} \delta-3,42 \frac{\bar{x}_{0} \bar{y}_{0}}{R_{0}}-10,7 \bar{x}_{0} \bar{q}_{0} \\
& -3,34 \bar{y}_{0} \bar{p}_{0}-8,48 R_{0} \bar{p}_{0} \bar{q}_{0} \\
\bar{q}_{5}= & -0,843 \frac{\bar{y}_{0}}{R_{0}}-1,457 \bar{q}_{0}+4 \frac{\bar{y}_{0} \delta}{R_{0}}+11,9 \bar{q}_{0} \delta-1,42 \frac{\bar{x}_{0} \bar{y}_{0}}{R_{0}^{2}}-5,83 \frac{\bar{x}_{0} \bar{q}_{0}}{R_{0}} \\
& -0,531 \frac{\bar{y}_{0} \bar{p}_{0}}{R_{0}}-3,32 \bar{p}_{0} \bar{q}_{0} .
\end{aligned}
$$

Si on combine l'action des deux prismes avec celle du miroir, on obtiendra les caractéristiques d'une trajectoire dans le plan de la pupille de sortie du système complet, en fonction des caractéristiques $x_{0}, y_{0}, p_{0}, q_{0}$ de la trajectoire dans le plan de la pupille d'entrée C.
Compte tenu des calculs de l'appendice II, nous obtenons :

- dans le cas du miroir convexe:

a) $\mathrm{Si}$ le sommet $\mathrm{S}$ du miroir est au point stigmatique $\mathrm{P}^{\prime}$ :

$$
\begin{aligned}
\bar{x}_{5}= & x_{0}+0 R_{0} \delta-4,29 x_{0} \delta-14,1 R_{0} p_{0} \delta-0,570 R_{0} \delta^{2}-0,660 \frac{x_{0}^{2}}{R_{0}}- \\
& -1,64 x_{0} p_{0}+2,88 \frac{y_{0}^{2}}{R_{0}}+7,16 y_{0} q_{0} \\
\bar{p}_{5}= & 0,806 \frac{x_{0}}{R_{0}}+p_{0}-0,0835 \delta-1,57 \frac{x_{0} \delta}{R_{0}}-5,09 p_{0} \delta-0,730 \delta^{2}-0,155 \frac{x_{0}^{2}}{R_{0}^{2}}+ \\
& +0,819 p_{0}^{2}+1,30 \frac{y_{0}^{2}}{R_{0}^{2}}+2,76 \frac{y_{0} q_{0}}{R_{0}}-0,158 q_{0}^{2} \\
\bar{y}_{5}= & -y_{0}+2,76 y_{0} \delta+6,88 R_{0} q_{0} \delta+2,14 \frac{x_{0} y_{0}}{R_{0}}+5,05 x_{0} q_{0}+0,258 y_{0} p_{0} \\
\bar{q}_{5}= & -0,806 \frac{y_{0}}{R_{0}}-q_{0}+1,64 \frac{y_{0} \delta}{R_{0}}+2,79 q_{0} \delta+0,664 \frac{x_{0} y_{0}}{R_{0}^{2}}+1,93 \frac{x_{0} q_{0}}{R_{0}}-0,258 p_{0} q_{0} .
\end{aligned}
$$

Dans ce cas, la «pente radiale » dépend au premier ordre de $\delta=\Delta v / v_{0} ;$ il y aura donc de la dispersion dans tout plan autre que le plan de la pupille de sortie du système dispersif:

b) Si le sommet du miroir est sur la focale achromatique $\Omega$ :

$\bar{x}_{5}=x_{0}+0 R_{0} \delta-4,69 x_{0} \delta-15,4 R_{0} p_{0} \delta-1,25 R_{0} \delta^{2}-0,540 \frac{x_{0}^{2}}{R_{0}}-1,37 x_{0} p_{0}+2,18 \frac{y_{0}^{2}}{R_{0}}+6,77 y_{0} q_{0}$.

Cette expression ne comporte plus de terme en $\delta$, en $p_{0}^{2}$ et en $q_{0}^{2}$.

$$
\begin{aligned}
\bar{p}_{5}= & 0,787 \frac{x_{0}}{R_{0}}+p_{0}+0 R_{0} \delta-1,64 \frac{x_{0} \delta}{R_{0}}-5,41 p_{0} \delta-1,06 \delta^{2}-0,139 \frac{x_{0}^{2}}{R_{0}^{2}}+ \\
& +0,686 p_{0}^{2}+0,938 \frac{y_{0}^{2}}{R_{0}^{2}}+2,57 \frac{y_{0} q_{0}}{R_{0}}-0,135 q_{0}^{2} .
\end{aligned}
$$

Le terme en $\delta$ disparaissant également de l'expression de $\bar{p}_{5}$, cela signifie qu'il n'y a plus de dispersion pour cette position du miroir. Seules subsistent, du point de vue chromatisme, les aberrations du second ordre. Le terme croisé en $R_{0} p_{0} q_{0}$ a disparu de l'expression de $\bar{y}_{5}$.

$$
\begin{gathered}
\bar{y}_{5}=-y_{0}+2,55 y_{0} \delta+7,91 R_{0} q_{0} \delta+0,925 \frac{x_{0} y_{0}}{R_{0}}+4,83 x_{0} q_{0}-1,61 y_{0} p_{0} . \\
\bar{q}_{5}=-0,645 \frac{y_{0}}{R_{0}}-q_{0}+1,27 \frac{y_{0} \delta}{R_{0}}+2,55 q_{0} \delta+0,186 \frac{x_{0} y_{0}}{R_{0}^{2}}+2,19 \frac{x_{0} q_{0}}{R_{0}}+1,61 p_{0} q_{0} .
\end{gathered}
$$


Nous constatons ainsi qu'un certain nombre de termes du second ordre ont disparu : les aberrations correspondantes ont été compensées par la double traversée du prisme magnétique.

Cependant, comme le système n'est pas stigmatique pour les points de l'axe lorsque $\mathrm{S}$ est en $\Omega$, nous pouvons supposer que l'on place en $C^{\prime \prime}$ une lentille convergente qui corrige l'astigmatisme en donnant de la focale radiale $M_{r}^{\prime \prime}$ une image qui coïncide avec la focale axiale $M_{a}^{\prime \prime}$. Le stigmatisme est ainsi réalisé sur l'axe, mais non pour des points pris hors de l'axe.

Une trajectoire quelconque caractérisée en projection sur le plan radial par $\left[\bar{p}_{5}, \bar{x}_{5}\right]$ sera transformée en $\left[\bar{p}=\bar{p}_{5}-k \bar{x}_{5}, \bar{x}_{5}\right]$ où $k$ est une constante qui traduit l'action du stigmateur $\left(k=0,1415 / R_{0}\right)$. Alors :

$$
\begin{gathered}
\bar{p}=0,645 \frac{x_{0}}{R_{0}}+p_{0}+0 R_{0} \delta-0,062 \frac{x_{0}^{2}}{R_{0}^{2}}+0,194 \frac{x_{0} p_{0}}{R_{0}}+0,686 p_{0}^{2}+0,629 \frac{y_{0}^{2}}{R_{0}^{2}}+ \\
+1,62 \frac{y_{0} q_{0}}{R_{0}}-0,135 q_{0}^{2}-0,972 \frac{x_{0} \delta}{R_{0}}-3,23 p_{0} \delta-0,879 \delta^{2}
\end{gathered}
$$

dans le cas du miroir concave :

$$
\begin{aligned}
\bar{x}_{5}= & -x_{0}+9,373 R_{0} \delta+11,5 x_{0} \delta+23,1 R_{0} p_{0} \delta-11,4 R_{0} \delta^{2}-2,66 \frac{x_{0}^{2}}{R_{0}} \\
& -11,3 x_{0} p_{0}-14,1 R_{0} p_{0}^{2}-3,60 \frac{y_{0}^{2}}{R_{0}}+1,97 y_{0} q_{0}+2,44 R_{0} q_{0}^{2} \\
\bar{p}_{5}= & -0,806 \frac{x_{0}}{R_{0}}-p_{0}+3,775 \delta+3,19 \frac{x_{0} \delta}{R_{0}}+5,06 p_{0} \delta-5,96 \delta^{2}-0,766 \frac{x_{0}^{2}}{R_{0}^{2}} \\
& -3,80 \frac{x_{0} p_{0}}{R_{0}}-5,66 p_{0}^{2}-1,31 \frac{y_{0}^{2}}{R_{0}^{2}}+1,15 \frac{y_{0} q_{0}}{R_{0}}+0,985 q_{0}^{2} \\
\bar{y}_{5}= & y_{0}-7,66 y_{0} \delta-31,6 R_{0} q_{0} \delta+3,15 \frac{x_{0} y_{0}}{R_{0}}+9,38 x_{0} q_{0}+9,38 y_{0} p_{0}+23,2 R_{0} p_{0} q_{0} \\
\bar{q}_{5}= & 0,806 \frac{y_{0}}{R_{0}}+q_{0}-3,74 \frac{y_{0} \delta}{R_{0}}-17,8 q_{0} \delta-0,518 \frac{x_{0} y_{0}}{R_{0}^{2}}+4,86 \frac{x_{0} q_{0}}{R_{0}}+11,2 \frac{y_{0} p_{0}}{R_{0}}+9,38 p_{0} q_{0} .
\end{aligned}
$$

Dans le cas particulier du prisme symétrique (angles d'incidence et d'émergence égaux et tels que $\operatorname{tg} \varepsilon=0,5$, positions des cross-over à $2 R_{0}$ avant la face d'entrée et au-delà de la face de sortie), le calcul géométrique des trajectoires donne (cf. [3]) :

$$
\begin{aligned}
x_{5}= & -x_{0}+4 R_{0} \delta+\frac{9}{4} x_{0} \delta+6 R_{0} p_{0} \delta-\frac{3}{2} R_{0} \delta^{2}-\frac{3}{4} \frac{x_{0}^{2}}{R_{0}}-3 x_{0} p_{0}-4 R_{0} p_{0}^{2} \\
& +\frac{y_{0}^{2}}{R_{0}}\left[\frac{1}{2}\left(1-\frac{\pi}{4}\right)^{2}-\frac{\pi}{4}\right]+y_{0} q_{0}\left(4-\frac{\pi}{2}\right)+4 R_{0} q_{0}^{2} \\
p_{5}= & -\frac{3 x_{0}}{4 R_{0}}-p_{0}+\frac{3}{2} \delta+\frac{9}{16} \frac{x_{0} \delta}{R_{0}}+\frac{5}{4} p_{0} \delta-\frac{9}{16} \delta^{2}-\frac{9}{64} \frac{x_{0}^{2}}{R_{0}^{2}}-\frac{3}{4} \frac{x_{0} p_{0}}{R_{0}}-\frac{3}{2} p_{0}^{2} \\
& +\frac{y_{0}^{2}}{R_{0}}\left[\frac{3}{16}-\frac{\pi}{8}+\frac{1}{4}\left(1-\frac{\pi}{4}\right)^{2}\right]+\frac{y_{0} q_{0}}{R_{0}}\left(2-\frac{\pi}{4}\right)+\frac{3}{2} q_{0}^{2} \\
y_{5}= & -y_{0}+y_{0} \delta\left(\frac{21}{4}-\frac{9 \pi}{8}\right)+11 R_{0} q_{0} \delta+\frac{x_{0} y_{0}}{R_{0}}\left(-\frac{17}{8}+\frac{\pi}{2}\right)-\frac{9}{2} x_{0} q_{0}+y_{0} p_{0}\left(-6+\frac{3 \pi}{4}\right)-12 R_{0} p_{0} q_{0} \\
q_{5}= & -\frac{y_{0}}{2 R_{0}}\left(2-\frac{\pi}{4}\right)-q_{0}+\frac{y_{0} \delta}{R_{0}}\left(\frac{5}{2}-\frac{19 \pi}{32}\right)+q_{0} \delta\left(\frac{23-\pi}{4}\right)+\frac{x_{0} y_{0}}{R_{0}^{2}}\left(-\frac{3}{4}+\frac{15 \pi}{64}\right) \\
& +\frac{y_{0} p_{0}}{R_{0}}\left(-2+\frac{5 \pi}{8}\right)+p_{0} q_{0}\left(-6+\frac{3 \pi}{4}\right) .
\end{aligned}
$$


Compte tenu du correcteur d'astigmatisme, la pente radiale $p_{5}$ devient

$$
\begin{aligned}
p= & \frac{\pi-8}{8 R_{0}} x_{0}-p_{0}+\frac{5-\pi}{2} \delta+\frac{9}{8}\left(1-\frac{\pi}{4}\right) \frac{x_{0} \delta}{R_{0}}+\frac{11-3 \pi}{4} p_{0} \delta+\frac{3 \pi-15}{16} \delta^{2}+\left(\frac{3 \pi}{32}-\frac{17}{64}\right) \frac{x_{0}^{2}}{R_{0}^{2}}- \\
& -\frac{3}{2}\left(1-\frac{\pi}{4}\right) \frac{x_{0} p_{0}}{R_{0}}+\frac{\pi-5}{2} p_{0}^{2}+\frac{y_{0}^{2}}{16 R_{0}^{2}}\left(9-7 \pi+\frac{11 \pi^{2}}{2}-\frac{\pi^{3}}{16}\right)+\frac{y_{0} q_{0}}{R_{0}}\left(3-\frac{7 \pi}{8}+\frac{\pi^{2}}{16}\right) \\
& +\frac{5-\pi}{2} q_{0}^{2} .
\end{aligned}
$$

Nous pouvons maintenant introduire, à la place des pentes $p_{0}$ et $q_{0}$ d'un rayon passant par un point $\mathrm{E}$ de l'objet, les pentes $\alpha$ et $\beta$ du rayon issu du centre $\mathrm{C}$ de la pupille d'entrée du prisme et passant par E (axe du pinceau) ainsi que $\lambda R_{0}$, distance de la pupille d'entrée au plan I de l'objet. Les caractéristiques d'une trajectoire dans le plan de la pupille de sortie $\mathrm{C}^{\prime \prime}$ deviennent des fonctions de $\lambda$ et permettent de déterminer les coordonnées $\bar{x}$ et $\bar{y}$ de l'intersection de la trajectoire avec un plan situé à la distance $Z_{0}$ de $\mathrm{C}^{\prime \prime}: \bar{x}=\bar{p}_{5} Z_{0}+\bar{x}_{5}$ et $\bar{y}=\bar{q}_{5} Z_{0}+\bar{y}_{5}$ et d'étudier leurs variations avec la position de l'objet.

ABERRATIONS INTRODUITES PAR LA PREMIÈRE TRAVERSÉE DU PRISME. - Il est intéressant d'examiner les aberrations qui affectent la pupille intermédiaire $\mathrm{C}^{\prime}$, car c'est dans le plan de C' que l'on place le diaphragme de sélection des bandes d'énergie.

Comme les aberrations prépondérantes au crossover $C^{\prime}$ sont dues à la distorsion [3], nous nous bornerons à l'examen des déformations introduites sur $C^{\prime}$ par les termes en $\alpha^{2}, \beta^{2}, \alpha \beta$. D'après les formules (I.7)

$$
\begin{array}{r}
x\left(\mathbf{C}^{\prime}\right)=-0,5 x_{0}-3,52 R_{0} \alpha^{2}+0,61 R_{0} \beta^{2}= \\
=-0,5 x_{0}+\Delta x \\
y\left(\mathbf{C}^{\prime}\right)=-1,46 y_{0}-17 R_{0} \alpha \beta= \\
=-1,46 y_{0}+\Delta y .
\end{array}
$$

$\mathrm{Au}$ premier ordre, l'image du diaphragme circulaire de rayon $r_{0}$ placé en $C$ est une ellipse car le grandissement est de $-0,5$ pour la première section principale et de $-1,46$ pour la deuxième. Le grand axe de l'ellipse est donc perpendiculaire au plan radial (pour $r_{0}=200 \mu$, le demi-grand axe est de $290 \mu$ et le demipetit axe de $100 \mu$ ).

Si on pose $\alpha=u \cos v$ et $\beta=u \sin v$ où $u$ est l'angle sous lequel on voit $\mathrm{du}$ point $\mathrm{C}$ le rayon $\mathrm{du}$ champ objet du prisme (champ supposé circulaire dans le plan I), le calcul montre que chaque point de l'image de la pupille d'entrée est entouré d'une aberration elliptique dont les demi-axes sont $2,1 \quad R_{0} u^{2}$ suivant $\mathrm{C}^{\prime} x$ et $8,5 R_{0} u^{2}$ le long de $\mathrm{C}^{\prime} y, u$ pouvant varier de 0 à sa valeur maximum $u_{0}$ correspondant à la limite du champ objet. Numériquement avec les données utilisées plus haut, l'ellipse a un petit axe de 19,5 $\mu$ et un grand axe vertical de $80 \mu$ (Fig. I.4).

L'image de la pupille d'entrée est donc déformée : elle est légèrement décalée vers les $\mathrm{x}$ négatifs, mais en outre, elle est encore étirée dans la direction des $y$.

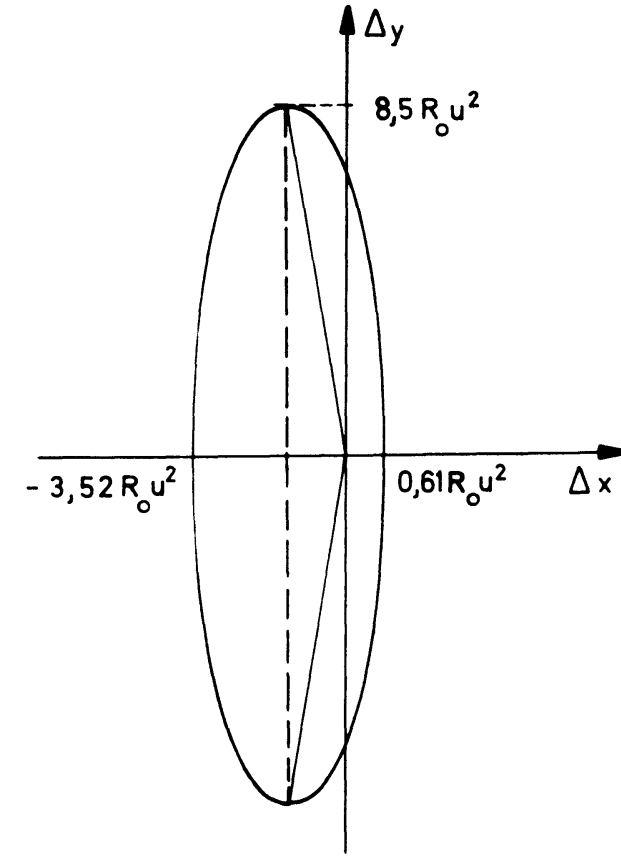

FIG. I.4. - Figure d'aberration de distorsion au cross-over intermédiaire $\mathrm{C}^{\prime}$.

Au total, le long de $y$, l'image a une longueur de $660 \mu$ et le long de l'axe $x$, une largeur de $220 \mu$.

Nous pouvons mentionner également les aberrations de champ introduites au niveau de l'image intermédiaire $I^{\prime}$ afin de mettre en évidence l'amélioration apportée par la double traversée du prisme. Nous examinons les résultats pour les deux positions intéressantes de l'objet: $\lambda=2,483$ et $\lambda=2,82$.

Lorsque le plan I est en $\mathrm{P}(\lambda=2,483)$, un pinceau plat du plan radial issu du diamètre de la pupille d'entrée et qui irait converger en un point du plan objet semble provenir, à la sortie du prisme, d'un point d'abscisse

$Z_{\mathrm{r}}=-\frac{\pi+2}{2} R_{0}(1+3,93 \alpha)=-2,571 R_{0}(1+3,93 \alpha)$

(comptée à partir de $\mathrm{C}^{\prime}$ ).

L'image d'un segment de droite du plan radial, perpendiculaire à l'axe $\mathrm{CO}$ en $\mathrm{P}$ est un segment de droite incliné sur 1'axe $x$ d'un angle $\varphi_{r}=-63^{\circ}$.

Pour un pinceau plat d'une deuxième section correspondant à un angle $\alpha$, le segment de droite image a pour équation

$$
Z_{\mathrm{a}}=-2,571 R_{0}(1+2,75 \alpha)
$$


et son inclinaison sur l'axe $\mathrm{x}$ est de $\varphi_{\mathrm{a}}=-54^{\circ}$.

Lorsque le plan objet I est perpendiculaire à l'axe $z$ au point $\mathrm{M}$ d'abscisse $\mathrm{CM}=\lambda R_{0}=2,82 R_{0}$, il n'y a plus stigmatisme sur l'axe lors de la première traversée. Si on étudie l'aspect à la sortie, de pinceaux plats contenus dans le plan radial et dans la seconde section correspondant à un angle $\alpha$ et qui convergent dans le plan I, les pinceaux émergents semblent provenir respectivement de points d'abscisses

et

$$
\begin{aligned}
& Z_{\mathrm{r}}=-1,72 R_{0}(1+2,29 \alpha) \\
& Z_{\mathrm{a}}=-2,42 R_{0}(1+2,60 \alpha) .
\end{aligned}
$$

Les angles d'inclinaison par rapport à la normale à $\mathrm{O}^{\prime} \mathrm{C}^{\prime}$ sont $\varphi_{\mathrm{r}} \simeq-49^{\circ}$ et $\varphi_{\mathrm{a}}=-52^{\circ} 30^{\prime}$.

Les plans $\Pi_{\mathrm{r}}$ et $\Pi_{\mathrm{a}}$, lieux des focales données par la première traversée, perpendiculaires au plan de symétrie, font un angle très faible entre eux, mais ils sont nettement inclinés sur l'axe optique et le défaut de champ introduit, sans compter l'astigmatisme sur l'axe, serait notable. La seconde traversée du prisme compense en grande partie ces défauts.

Appendice II. - ACTION DU MIROIR SUR LES TRAJECTOIRES (approximation de Gauss). - On suppose que le miroir n'introduit aucune aberration sur les trajectoires, et on détermine les matrices de passage.

\section{Cas du miroir convexe.}

Le centre du miroir est placé en $C^{\prime}$ et le rayon du miroir est égal à $\mathrm{r} R_{0}$ ( $\mathrm{r}$ nombre sans dimension).

Soit un rayon incident AM issu du point $\mathrm{A}$ de la « surface » du miroir, qui traverse le plan du cross-over au point $\mathrm{M}$ d'abscisse $x$ (Fig. II.1). Sa pente est $p$. Le rayon réfléchi correspondant, $\mathrm{M}_{\mathrm{m}} \mathrm{A}$, traverse le plan du cross-over au point $M_{m}$ d'abscisse $x_{m}$ et sa pente est $p_{\mathrm{m}}$. On a :

$$
\begin{aligned}
& x_{\mathrm{m}}=-x \\
& p_{\mathrm{m}}=\frac{h-x+x_{\mathrm{m}}}{\mathrm{r} R_{0}}=p-\frac{2 x}{\mathrm{r} R_{0}}
\end{aligned}
$$

avec

$$
p=\frac{h}{\mathrm{r} R_{0}}\left(\text { au } 2^{\mathrm{e}} \text { ordre près }\right)
$$

ou

$$
\begin{aligned}
& {\left[\begin{array}{l}
\bar{x}_{0} \\
\bar{p}_{0}
\end{array}\right]=\left[\begin{array}{rr}
-1 & 0 \\
0 & 1
\end{array}\right]\left[\begin{array}{l}
-1 \\
-\frac{2}{r R_{0}}
\end{array}\right.} \\
& {\left[\begin{array}{l}
\bar{y}_{0} \\
\bar{q}_{0}
\end{array}\right]=\left[\begin{array}{rr}
1 & 0 \\
0 & -1
\end{array}\right]\left[\begin{array}{l}
-1 \\
-\frac{2}{\mathrm{r} R_{0}}
\end{array}\right.}
\end{aligned}
$$



FIG. II.1. - Miroir convexe centré en $\mathbf{C}^{\prime}$; AS « surface réfléchissante ».

d'où, pour la première section principale

$$
\left[\begin{array}{l}
x_{\mathrm{m}} \\
p_{\mathrm{m}}
\end{array}\right]=\left[\begin{array}{ll}
-1 & 0 \\
-\frac{2}{r \mathrm{R}_{0}} & 1
\end{array}\right]\left[\begin{array}{l}
x \\
p
\end{array}\right] .
$$

On obtiendrait de même, pour la seconde section principale :

$$
\left[\begin{array}{l}
y_{\mathrm{m}} \\
q_{\mathrm{m}}
\end{array}\right]=\left[\begin{array}{ll}
-1 & 0 \\
-\frac{2}{\mathrm{r} R_{0}} & 1
\end{array}\right]\left[\begin{array}{l}
y \\
q
\end{array}\right] .
$$

Pour que les formules relatives aux deux traversées du prisme puissent être déterminées de manière analogue, il faut que l'axe des $x$ soit toujours orienté vers l'extérieur de la trajectoire. Donc, à la réflexion du faisceau sur le miroir doit correspondre une réflexion des axes $x$ et $z$, l'axe y restant inchangé pour que le nouveau trièdre soit encore un trièdre direct. Dans ces conditions, $x_{\mathrm{m}}$ est changé en $\bar{x}_{0}=-x_{\mathrm{m}}$ et $p_{\mathrm{m}}$ en $\bar{p}_{0}=p_{\mathrm{m}}, \bar{y}_{0}=y_{\mathrm{m}}$ et $\bar{q}_{0}=-q_{\mathrm{m}}$ car l'axe $\mathrm{z}$ est aussi inversé ; ceci peut se mettre sous forme matricielle. Les matrices qui décrivent la réflexion des trajectoires et des axes de référence, et qui feront donc correspondre aux coordonnées dans le plan du cross-over de sortie lors de la première traversée les coordonnées $\bar{x}_{0}, \bar{y}_{0}, \bar{p}_{0}, \bar{q}_{0}$ dans le plan du cross-over d'entrée lors de la seconde traversée seront :

$$
\begin{aligned}
& \left.\begin{array}{l}
0 \\
1
\end{array}\right]\left[\begin{array}{l}
x \\
p
\end{array}\right]=\left[\begin{array}{cc}
1 & 0 \\
-\frac{2}{r R_{0}} & 1
\end{array}\right]\left[\begin{array}{l}
x \\
p
\end{array}\right] \\
& \left.\begin{array}{l}
0 \\
1
\end{array}\right]\left[\begin{array}{l}
y \\
q
\end{array}\right]=\left[\begin{array}{rr}
-1 & 0 \\
\frac{2}{\mathrm{r} R_{0}} & -1
\end{array}\right]\left[\begin{array}{l}
y \\
q
\end{array}\right]
\end{aligned}
$$

$$
\bar{x}_{0}=x, \bar{p}_{0}=p-\frac{2 x}{\mathrm{r} R_{0}}, \quad \bar{y}_{0}=-y, \bar{q}_{0}=-q+\frac{2 y}{\mathrm{r} R_{0}} .
$$

virtuel $\mathbf{P}^{\prime}$. Le plan du cross-over correspond cette fois à la «surface » du miroir (Fig. II.2). En prenant les mêmes notations que pour le miroir convexe, on obtient pour un rayon quelconque :
Le sommet $\mathrm{S}$ du miroir est au cross-over $\mathrm{C}^{\prime}$ et le centre $\Gamma \mathrm{du}$ miroir est sur le point stigmatique image

\section{Cas du miroir concave.}




$$
\begin{aligned}
& x_{\mathrm{m}}=x \\
& p_{\mathrm{m}}=\frac{2 x}{\mathrm{r} R_{0}}-p \text { avec } \mathrm{S} \Gamma=\mathrm{r} R_{0} .
\end{aligned}
$$

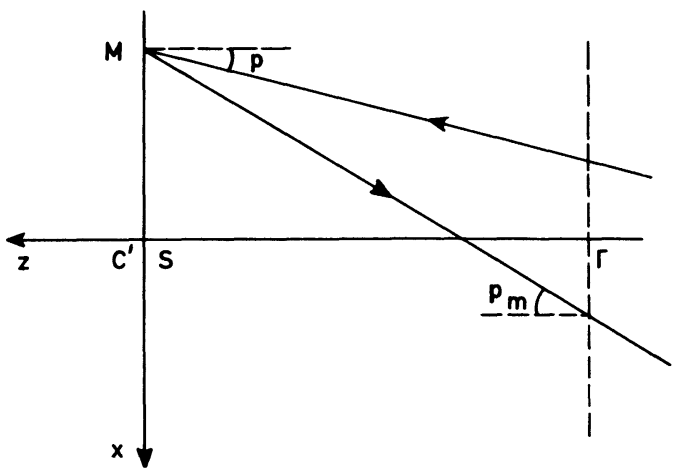

FIG. II.2. - Miroir concave centré en $\Gamma: \mathrm{C}^{\prime} \mathrm{S}$ «surface» du miroir.
En tenant compte du changement d'axes $x, z$, les coordonnées après réflexion sur le miroir sont $\bar{x}_{0}$ et $\bar{p}_{0}$ telles que :

$\bar{x}_{0}=-x \quad$ (première section principale)

$\bar{p}_{0}=\frac{2 x}{\mathrm{r} R_{0}}-p$

$\bar{y}_{0}=y$

$\bar{q}_{0}=-\frac{2 y}{\mathrm{r} R_{0}}+q \quad$ (deuxième section principale).

L'action du miroir sur les trajectoires émergeant du premier prisme lors de la première traversée transforme les coordonnées $x_{5}, y_{5}, p_{5}, q_{5}$ en $\bar{x}_{0}, \bar{y}_{0}, \bar{p}_{0}, \bar{q}_{0}$.

- Cas du miroir convexe :

a) si le sommet du miroir est sur la focale achromatique $\Omega(r=2,343)$.

$$
\begin{aligned}
\bar{x}_{0}= & x_{5}, \bar{y}_{0}=y_{5} \\
\bar{p}_{0}= & -0,573 \frac{x_{0}}{R_{0}}-2 p_{0}-\delta-0,844 \frac{x_{0} \delta}{R_{0}}-2,56 p_{0} \delta+0,427 \delta^{2}+0,427 \frac{x_{0}^{2}}{R_{0}^{2}} \\
& +1,22 \frac{x_{0} p_{0}}{R_{0}}+0,814 p_{0}^{2}+0,756 \frac{y_{0}^{2}}{R_{0}^{2}}+1,09 \frac{y_{0} q_{0}}{R_{0}}-0,124 q_{0}^{2} \\
\bar{q}_{0}= & -0,400 \frac{y_{0}}{R_{0}}+0,686 q_{0}+0,796 \frac{y_{0} \delta}{R_{0}}+2,31 q_{0} \delta-0,673 \frac{x_{0} y_{0}}{R_{0}^{2}}-1,06 \frac{x_{0} y_{0}}{R_{0}}-4,61 \frac{y_{0} p_{0}}{R_{0}}-7,80 p_{0} q_{0}
\end{aligned}
$$

b) si le sommet du miroir est sur le point stigmatique $\mathrm{P}^{\prime}$, le rayon est

$$
\begin{aligned}
r= & \frac{\pi+2}{2}=2,571 \\
\bar{x}_{0}= & x_{5}, \bar{y}_{0}=-y_{5} \\
\bar{p}_{0}= & -0,611 \frac{x_{0}}{R_{0}}-2 p_{0}-0,823 \delta-0,778 x_{0} \delta-2,33 p_{0} \delta+0,389 \delta^{2}+0,389 \frac{x_{0}^{2}}{R_{0}^{2}} \\
& +1,04 \frac{x_{0} p_{0}}{R_{0}}+0,548 p_{0}^{2}+0,633 \frac{y_{0}^{2}}{R_{0}^{2}}+0,988 \frac{y_{0} q_{0}}{R_{0}}-0,078 q_{0}^{2} \\
\bar{q}_{0}= & -0,290 \frac{y_{0}}{R_{0}}+0,686 q_{0}+0,679 \frac{y_{0} \delta}{R_{0}}+1,87 q_{0} \delta-0,642 \frac{x_{0} y_{0}}{R_{0}^{2}}-0,821 \frac{x_{0} q_{0}}{R_{0}} \\
& -4,11 \frac{y_{0} p_{0}}{R_{0}}-6,51 p_{0} q_{0} .
\end{aligned}
$$

- Cas du miroir concave $(r=(\pi+2) / 2)$.

Les valeurs des coordonnées et des pentes sont les opposées de celles données par les équations (II.4).

\section{Bibliographie}

[1] CASTAing (R.), IVe Congrès international sur l'Optique des Rayons X et la Microanalyse, Orsay, 1965, p. 48, Hermann, Paris.

[2] Castaing (R.), Jouffrey (B.), Slodzian (G.), $C . R$. Acad. Sci., Paris, 1960, 251, 1010.
[3] Slodzian (G.), Thèse de Doctorat, 1963, Paris ; Ann. de Phys., 1964, (13), 9, 591.

[4] Castaing (R.), Slodzian (G.), Advances in Mass Spectrometry, 1965, 3, 91, Pergamon Press.

[5] Castaing (R.), Henry (L.), J. Microscopie, 1964, 3,133. 
[6] Henry (L.), Thèse de Doctorat, 1964, Paris ; Bull. Soc. Franç. Minéral. Cris., 1965, 88, 172.

[7] Castaing (R.), Hennequin (J.-F.), HenRy (L.), SLodzian (G.), dans Focusing of charged particles, 2, éd. Septier (A.), 1967, Academic Press, New York.

[8] Slodzian (G.), Rev. Phys. Appl., 1968, 3, 360.

[9] Cotte (M.), Thèse de Doctorat, 1938, Paris ; Ann. de Phys., 1938, (11), 10, 333.

[10] Cross (W. G.), Rev. Sci. Instr., 1951, 22, 717.

[11] Rouberol (J. M.), communication privée.
[12] Hennequin (J.-F.), Diplôme d'Etudes Supérieures, 1960, Paris.

[13] WollnIK (H.), Nucl. Instr. Meth., 1965, 34, 213 ; Nucl. Instr. Meth., 1967, 52, 250 ; Nucl. Instr. Meth., 1967, 53, 197.

[14] Enge (H. A.), dans Focusing of charged particles, 2, éd. Septier (A.), 1967, Academic Press, New York.

[15] ZwORYKIN (V. K.) et coll., Electron Optics and the Electron Microscope, 1946, J. Wiley and Sons, New York. 